\title{
zCOSMOS - 10k-bright spectroscopic sample
}

\section{The bimodality in the galaxy stellar mass function: exploring its evolution with redshift ${ }^{\star}$}

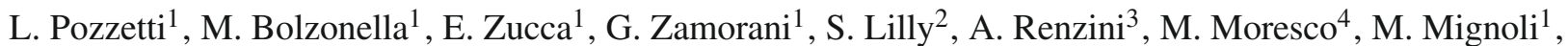 \\ P. Cassata ${ }^{5,6}$, L. Tasca ${ }^{7,6}$, F. Lamareille ${ }^{8}$, C. Maier ${ }^{2}$, B. Meneux ${ }^{9,10}$, C. Halliday ${ }^{11}$, P. Oesch ${ }^{2}$, D. Vergani ${ }^{1}$, K. Caputi ${ }^{2}$, \\ K. Kovač ${ }^{2}$, A. Cimatti ${ }^{4}$, O. Cucciati ${ }^{6,12}$, A. Iovino ${ }^{12}$, Y. Peng ${ }^{2}$, M. Carollo ${ }^{2}$, T. Contini ${ }^{8}$, J.-P. Kneib ${ }^{6}$, O. Le Févre ${ }^{6}$, \\ V. Mainieri ${ }^{9}$, M. Scodeggio ${ }^{7}$, S. Bardelli ${ }^{1}$, A. Bongiorno ${ }^{9}$, G. Coppa $^{1}$, S. de la Torre ${ }^{6}$, L. de Ravel ${ }^{6}$, P. Franzetti $^{7}$, \\ B. Garilli ${ }^{7}$, P. Kampczyk ${ }^{2}$, C. Knobel ${ }^{2}$, J.-F. Le Borgne ${ }^{8}$, V. Le Brun ${ }^{6}$, R. Pellò ${ }^{8}$, E. Perez Montero ${ }^{8}$, E. Ricciardelli ${ }^{3}$, \\ J. D. Silverman ${ }^{2}$, M. Tanaka ${ }^{9}$, L. Tresse ${ }^{6}$, U. Abbas ${ }^{6}$, D. Bottini ${ }^{7}$, A. Cappi ${ }^{1}$, L. Guzzo ${ }^{12}$, A. M. Koekemoer ${ }^{13}$, \\ A. Leauthaud ${ }^{6}$, D. Maccagni ${ }^{7}$, C. Marinoni ${ }^{14}$, H. J. McCracken ${ }^{15}$, P. Memeo ${ }^{7}$, C. Porciani ${ }^{2}$, R. Scaramella ${ }^{16}$, \\ C. Scarlata ${ }^{17}$, and N. Scoville ${ }^{18}$
}

(Affiliations can be found after the references)

Received 30 July 2009 / Accepted 31 May 2010

\begin{abstract}
We present the galaxy stellar mass function $(G S M F)$ to redshift $z \simeq 1$, based on the analysis of about 8500 galaxies with $I<22.5$ (AB mag) over $1.4 \mathrm{deg}^{2}$, which are part of the zCOSMOS-bright 10k spectroscopic sample. We investigate the total GSMF, as well as the contributions of early- and late-type galaxies (ETGs and LTGs, respectively), defined by different criteria (broad-band spectral energy distribution, morphology, spectral properties, or star formation activities). We unveil a galaxy bimodality in the global GSMF, whose shape is more accurately represented by 2 Schechter functions, one linked to the ETG and the other to the LTG populations. For the global population, we confirm a mass-dependent evolution ("mass-assembly downsizing"), i.e., galaxy number density increases with cosmic time by a factor of two between $z=1$ and $z=0$ for intermediate-to-low mass $\left(\log \left(\mathcal{M} / \mathcal{M}_{\odot}\right) \sim 10.5\right)$ galaxies but less than $15 \%$ for $\log \left(\mathcal{M} / \mathcal{M}_{\odot}\right)>11$. We find that the $G S M F$ evolution at intermediateto-low values of $\mathcal{M}\left(\log \left(\mathcal{M} / \mathcal{M}_{\odot}\right)<10.6\right)$ is mostly explained by the growth in stellar mass driven by smoothly decreasing star formation activities, despite the redder colours predicted in particular at low redshift. The low residual evolution is consistent, on average, with $\sim 0.16$ merger per galaxy per Gyr (of which fewer than 0.1 are major), with a hint of a decrease with cosmic time but not a clear dependence on the mass. From the analysis of different galaxy types, we find that ETGs, regardless of the classification method, increase in number density with cosmic time more rapidly with decreasing $\mathcal{M}$, i.e., follow a top-down building history, with a median "building redshift" increasing with mass $(z>1 \mathrm{for}$ $\left.\log \left(\mathcal{M} / \mathcal{M}_{\odot}\right)>11\right)$, in contrast to hierarchical model predictions. For LTGs, we find that the number density of blue or spiral galaxies with $\log \left(\mathcal{M} / \mathcal{M}_{\odot}\right)>10$ remains almost constant with cosmic time from $z \sim 1$. Instead, the most extreme population of star-forming galaxies (with high specific star formation), at intermediate/high-mass, rapidly decreases in number density with cosmic time. Our data can be interpreted as a combination of different effects. Firstly, we suggest a transformation, driven mainly by SFH, from blue, active, spiral galaxies of intermediate mass to blue quiescent and subsequently (1-2 Gyr after) red, passive types of low specific star formation. We find an indication that the complete morphological transformation, probably driven by dynamical processes, into red spheroidal galaxies, occurred on longer timescales or followed after 1-2 Gyr. A continuous replacement of blue galaxies is expected to be accomplished by low-mass active spirals increasing their stellar mass. We estimate the growth rate in number and mass density of the red galaxies at different redshifts and masses. The corresponding fraction of blue galaxies that, at any given time, is transforming into red galaxies per Gyr, due to the quenching of their $S F R$, is on average $\sim 25 \%$ for $\log \left(\mathcal{M} / \mathcal{M}_{\odot}\right)<11$. We conclude that the build-up of galaxies and in particular of ETGs follows the same downsizing trend with mass (i.e. occurs earlier for high-mass galaxies) as the formation of their stars and follows the converse of the trend predicted by current SAMs. In this scenario, we expect there to be a negligible evolution of the galaxy baryonic mass function $(G B M F)$ for the global population at all masses and a decrease with cosmic time in the $G B M F$ for the blue galaxy population at intermediate-high masses.
\end{abstract}

Key words. galaxies: evolution - galaxies: luminosity function, mass function - galaxies: statistics - galaxies: formation

\section{Introduction}

Tracing the history of both galaxy star formation (Lilly et al. 1996; Madau et al. 1996, 1998) and stellar mass assembly (Dickinson et al. 2003) over cosmic time represent major challenges in modern cosmology. In particular, it is still uncertain how the bimodality seen in the local Universe in terms of galaxy properties (e.g., colour, morphology, star formation, and spectral

* Based on data obtained with the European Southern Observatory Very Large Telescope, Paranal, Chile, program 175.A-0839. features; Kauffmann et al. 2003; Baldry et al. 2004; Brinchmann et al. 2004) evolves and when it was created. Galaxies in the local Universe exhibit distinctive bimodal colour distributions (Strateva et al. 2001; Hogg et al. 2002; Blanton et al. 2003), which suggests that different evolution histories exist for galaxies lying on the two sequences (Menci et al. 2005; Scarlata et al. 2007b; De Lucia et al. 2007). Even if colour bimodality has already been observed and studied at higher redshift (Bell et al. 2004; Weiner et al. 2005, up to $z \sim 1$; Franzetti et al. 2007; Cirasuolo et al. 2007, up to $z \sim 1.5$; Giallongo et al. 2005; 
Cassata et al. 2008; Williams et al. 2009, up to $z \sim 2$ ), no study until now has fully evaluated how the number densities of the two populations compare and evolve with cosmic time.

It is still unclear, indeed, how the galaxy stellar mass function $(G S M F)$ is linked to the galaxy bimodality. Baldry et al. (2004, 2006, 2008) noted a bimodal shape in the local GSMF in the SDSS with an upturn at masses lower than $10^{9} \mathcal{M}_{\odot}$, related to the two different galaxy populations. It is, therefore, extremely interesting to explore how this shape evolves with redshift and which mechanisms contribute to its appearance. From previous surveys, it is well established that there has been an increase/decrease in the fraction of red/blue or early/late-type galaxies with cosmic time at intermediate masses since $z \sim 1$ (Fontana et al. 2004; Bundy et al. 2006; Arnouts et al. 2007; Scarlata et al. 2007b; Vergani et al. 2008), while the precise evolution in absolute number densities of the two populations remains controversial, in particular for intermediate/massive galaxies. A knowledge of this evolution may help to constrain the quenching mechanisms responsible for downsizing.

In agreement with the first formulation introduced by Cowie et al. (1996), a downsizing scenario in both age and star formation history has been proposed by several observational studies, i.e., more massive galaxies form their stars earlier and more rapidly than lower mass ones (hereafter age downsizing: Brinchmann \& Ellis 2000; Gavazzi \& Scodeggio 1996; Fontana et al. 2004; Kodama et al. 2004; Bauer et al. 2005; Feulner et al. 2005a,b; Juneau et al. 2005; Borch et al. 2006; Cucciati et al. 2006; Vergani et al. 2008). Age downsizing occurs separately for each of the two populations causing galaxy bimodality, i.e., a red peak (Thomas et al. 2005; Fontana et al. 2004; Thomas et al. 2009) and a blue one (Noeske et al. 2007a,b). However, it remains unclear whether the age downsizing is coupled with a mass-assembly downsizing scenario for galaxy evolution and formation (Fontana et al. 2004, 2006; Pozzetti et al. 2003, 2007; Cimatti et al. 2006; Bundy et al. 2006), i.e., if the more massive galaxies assembled their mass earlier than lower mass ones. Furthermore, do low-mass galaxies contain younger stars and assemble later even within the same spectral type? The hierarchical model of De Lucia et al. (2006) predicts, for example, a bottomup assembly history for elliptical galaxies (also called "upsizing") following the hierarchical growth of dark matter haloes, in contrast to a top-down, downsizing scenario for the formation of their stars.

A fundamental role towards answering these questions is played by deep surveys, which sample thousands of galaxies across large portions of the sky. Very deep surveys have been exploited to describe the shape of the stellar mass function at high redshift (Fontana et al. 2006; Drory et al. 2005; Gwyn \& Hartwick 2005; Bundy et al. 2006; Pozzetti et al. 2007), but a clear picture about stellar mass assembly and how it depends on mass (mass-assembly downsizing) and galaxy type has not yet emerged. Previous studies have explored the evolution of different galaxy types in deep near-IR surveys, such as K20, by means of the $K$-band luminosity function (Pozzetti et al. 2003) and the galaxy stellar mass function (Fontana et al. 2004), using spectral classification (i.e., absorption-line galaxies versus emissionline galaxies), and in larger optical and near-IR surveys such as VVDS, COMBO17, and DEEP2, using colours or spectra to define galaxy types (Bell et al. 2004; Cimatti et al. 2006; Faber et al. 2007; Zucca et al. 2006; Arnouts et al. 2007; Vergani et al. 2008). Even if the results of these surveys remain disputed (compare for example Bell et al. 2004; and Cimatti et al. 2006, for the same dataset), most of these studies agree that luminous and rather massive old galaxies were already quite common at $z \sim 1$ and that their number density declines rapidly at yet higher redshift. This suggests that merger events are ruled out as the major mechanism behind their assembly history below $z \simeq 1$. However, observational results about major merging and dry merging are still contradictory (see Bell et al. 2006; van Dokkum 2005; Lin et al. 2004; de Ravel et al. 2009; and Renzini 2007, for a summary). It is also known that the less luminous/massive ETGs decline in number density steadily with redshift (Cimatti et al. 2006). Scarlata et al. (2007b) show similar results for the photometric survey COSMOS, using both morphologically and photometrically selected subsamples of early-type galaxies. The evolution in number density of the most massive late-type starforming galaxies continues, however, to be unclear.

Another open question is whether the evolution of the observed GSMF with cosmic time is driven mainly by either merging events at any given mass, as predicted by hierarchical galaxy formation models (Cole et al. 2000; Menci et al. 2005; Bower et al. 2006; De Lucia et al. 2006; Monaco et al. 2006) or star formation histories (SFHs, see Vergani et al. 2008; Walcher et al. 2008). It is widely believed that galaxies are assembled by hierarchical mergers between massive cold dark matter haloes, in which baryonic star-forming matter is embedded. However, most of the hierarchical galaxy assembly models are unable to completely account for the observed GSMF and its evolution (see Fontana et al. 2004, 2006; Monaco et al. 2006; Caputi et al. 2006; Marchesini et al. 2009; Fontanot et al. 2009, for a detailed comparison with models). For instance, some models tend to underpredict the high-mass tail and overpredict the rate of its evolution (Fontana et al. 2004, 2006), even in the extreme case of evolution driven purely by mergers (Monaco et al. 2006). Only the assumption that a significant fraction, $\sim 30 \%$, of stars are scattered within the diffuse stellar component at each merger event leads to significant suppression of the predicted evolution rate, in closer agreement with observational constraints (Monaco et al. 2006). On the other hand, most of the models overpredict the number density of relatively low-mass galaxies (see Fontana et al. 2006; Kitzbichler \& White 2007; Marchesini et al. 2009; Fontanot et al. 2009). Furthermore, the models do not reproduce the downsizing trend in stellar mass observed for elliptical galazies (Cimatti et al. 2006). According to the latest observational results, the current galaxy formation and evolutionary scenario is becoming one in which a smoother evolution in mass growth and star formation (due to cold gas accretion) plays a major, if not dominant, role compared to dark matter (major) merging events.

In this paper, we use the zCOSMOS spectroscopic survey (Lilly et al. 2007, 2009) to complete a comprehensive analysis of the GSMF. Compared to previous spectroscopic surveys, the larger area and number of spectroscopic redshifts of zCOSMOS allows higher precision, higher quality statistics, and lower cosmic variance (Lilly et al. 2009) to be achieved in our analysis, in particular for the massive end of the GSMFs. We derive the $G S M F$ and its evolution with cosmic time since $z=1$, as well as the contribution of different galaxy populations, using numerous classification methods defined in terms of their colours, morphologies, star formation activities, or spectroscopic classifications. By using spectroscopic redshifts, we are able to study the shape of the global GSMF to high precision, its evolution, and how it is related to the bimodalities in galaxy properties (colours, morphologies, spectral properties) observed to high redshifts. We explore the different roles of merging and SFHs as a function of $\mathcal{M}$ and cosmic time to explain the observed GSMF evolution. We also explore the evolution with cosmic time of the GSMF for different galaxy types and propose an evolutionary scenario. 
Throughout the paper, we adopt the cosmology $\Omega_{\mathrm{m}}=0.25$ and $\Omega_{\Lambda}=0.75$, with $h_{70}=H_{0} /\left(70 \mathrm{~km} \mathrm{~s}^{-1} \mathrm{Mpc}^{-1}\right)$. Magnitudes are given in the AB system.

\section{COSMOS and the bright $10 \mathrm{k} z$ COSMOS spectroscopic sample}

The Cosmic Evolution Survey (COSMOS, Scoville et al. 2007) is the largest HST survey (640 orbits) ever undertaken, imaging a field of $\sim 2 \mathrm{deg}^{2}$ with the Advanced Camera for Surveys (ACS) with F814W (Koekemoer et al. 2007). This survey was designed to probe galaxy evolution and the effects of environment to high redshift. COSMOS observations include good coverage of the field with multiband photometry from the UV (with GALEX, Zamojski et al. 2007), optical (with Subaru and CFHT, Taniguchi et al. 2007; Capak et al. 2007), NIR (with CTIO, KPNO, Capak et al. 2007; and CFHT, McCracken et al. 2010), to MIR and FIR (S-COSMOS with Spitzer, Sanders et al. 2007), in combination with a multiwavelength dataset from radio (with VLA, Schinnerer et al. 2007), millimetre (with MAMBO-2 at the IRAM telescope, Bertoldi et al. 2007), to X-rays (with XMM, Hasinger et al. 2007; and Chandra, Elvis et al. 2009).

The zCOSMOS spectroscopic survey (Lilly et al. 2007) is an ongoing ESO Large Programme ( $600 \mathrm{~h}$ of observations) aiming to map the COSMOS field with the VIsible Multi-Object Spectrograph (VIMOS, Le Fèvre et al. 2003a), mounted on the ESO Very Large Telescope (VLT). The zCOSMOS survey consists of a bright part, with spectroscopy limited to objects in the magnitude range $15.0<I<22.5$, and of a deep part, which measures redshifts of $B<25.25$ of galaxies colour-selected to be in the range $1.4<z<3$, within the central $1 \mathrm{deg}^{2}$. The bright part has already produced redshifts and spectra for about 10000 galaxies over $\sim 1.4 \mathrm{deg}^{2}$, the so-called 10k-bright spectroscopic sample (Lilly et al. 2009), with an average sampling rate of about $33 \%$. For more details about the zCOSMOS 10kbright sample, we refer to Lilly et al. (2009). Here we recall that the VIMOS spectroscopic observations were completed using the red medium resolution $R \sim 600$ MR grism (5550-9650 $\AA$ ), the spectra were reduced using the VIMOS Interactive Pipeline Graphical Interface software (VIPGI, Scodeggio et al. 2005) and redshift measurements were visually determined after a first estimate had been provided by an automatic package (EZ, Garilli et al., in prep.). A quality flag was assigned to each redshift measurement. This flag ranges from 0 (failed measurement) to 4 ( $100 \%$ confidence level), and flag 9 indicates spectra with a single emission line, for which multiple redshift solutions are possible. Additional details about the spectroscopic quality flags and their probability of being robust are given in Lilly et al. (2009, see their Table 1). In addition to the confidence classes described in Lilly et al. (2007), a decimal place (from 5 to 1, see Table 3 in Lilly et al. 2009) in the class is added to indicate the level of consistency between the spectroscopic and photometric redshifts obtained by the Zurich Extragalactic Bayesian Redshift Analyzer (ZEBRA; Feldmann et al. 2006), using the optical to infrared SED.

\subsection{The selected galaxy spectroscopic sample}

The analysis presented in this paper is based on the zCOSMOS10k bright sample (Lilly et al. 2009). From the total sample of 10644 objects observed spectroscopically, we used the objects within the statistical sample defined in the magnitude range $15<$ $I<22.5$, and removed the spectroscopically confirmed stars, broad-line AGNs, and the galaxies with low quality redshift flag (flag $<1.5$, i.e., with a verification rate $<90 \%$ and a spectroscopic redshift inconsistent with the photometric redshift). To achieve reliable SED fitting to multi-band photometric data, we excluded objects with apparent magnitudes measured in fewer than 5 bands $(\sim 1.7 \%)$ and objects for which the ground photometry can be affected by the blending of sources, as inferred from the number of ACS sources brighter than $I=22.5$ within $0.6^{\prime \prime}$ $(\sim 0.5 \%)$. This resulted in the selection of 8450 galaxy spectra with secure spectroscopic measurements (7936 in the redshift range where the following analysis is carried out, $z=0.1-1$ ) over $5045.80 \mathrm{arcmin}^{2}$. Objects with redshift flags $<1.5$ are taken into account statistically (see Sect. 5, and Bolzonella et al. 2009; and Zucca et al. 2009, for details).

The final selected sample of 8450 galaxies, which have highly reliable spectroscopic redshifts, have complete multiband photometric coverage (from UV to IRAC) and morphological classifications, along with morphological parameters from both the Zurich Estimator of Structural Types (ZEST; Scarlata et al. 2007a) and estimates obtained in Marseille (Cassata et al. 2008; Tasca et al. 2009, MRS hereafter).

\subsection{Photometric data}

The COSMOS field has been covered by multiband photometry over a wide range of wavelengths. In this paper, we used the observed magnitudes in 10 photometric bands (CFHT $u^{*}$ and $K_{\mathrm{s}}$, Subaru $B_{J}, V_{J}, g^{+}, r^{+}, i^{+}$, and $z^{+}$, and Spitzer IRAC at $3.6 \mu \mathrm{m}$ and $4.5 \mu \mathrm{m})$. Descriptions of the photometric catalogs are given in Capak et al. (2007), Sanders et al. (2007), and McCracken et al. (2010). Following the same approach as Capak et al. (2007, see their Table 13), the photometry was adjusted by applying fixed zeropoint offsets to the observed magnitudes in each band to statistically reduce the differences between observed and reference magnitudes computed from a set of template SEDs, finding in general for each band very similar offsets.

\section{Estimate of the stellar masses}

We used the stellar masses $(\mathcal{M})$ estimated from a fit to the multicolour spectral energy distribution (SED), using the observed magnitudes in 10 photometric bands from $u^{*}$ to $4.5 \mu \mathrm{m}$ (see Sect. 2.2), and following the method described in Pozzetti et al. (2007). In a parallel paper, Bolzonella et al. (2009) describe the different methods used to compute stellar masses, based on different assumptions about the population synthesis models (Bruzual \& Charlot 2003, BC03; Maraston 2005, M05; Charlot \& Bruzual 2007, CB07) and the star-formation histories (SFHs), such as smooth exponentially decreasing or complex SFHs with the addition of secondary bursts. In Bolzonella et al., the associated uncertainties and degeneracies are also discussed (see also Fontana et al. 2004; Pozzetti et al. 2007; Marchesini et al. 2009). Here we just recall that the accuracy of the photometric stellar masses is satisfactory overall, with typical dispersions caused by statistical uncertainties and degeneracies of the order of 0.2 dex. We note that the addition of secondary bursts to a continuous star-formation history produces systematically higher (up to $40 \%$ on average) stellar masses (Pozzetti et al. 2007), and in particular Fontana et al. (2004) demostrated that the effect is larger when the fraction of mass produced in the burst is small. On the other hand, population synthesis models with a TP-AGB phase (Maraston 2005; Charlot \& Bruzual 2007) produce shifts of up to $\sim 0.2$ dex towards lower $\mathcal{M}$. Finally, the uncertainty in the absolute value of the $\mathcal{M}$ related to the assumptions about the 
initial mass function (IMF) is within a factor of 2 for the typical $I M F$ s usually adopted in the literature.

In this paper, we adopt stellar masses derived with Hyperzmass (see Pozzetti et al. 2007), a modified version of the photometric redshift code Hyperz (Bolzonella et al. 2000). We used smooth exponentially decreasing SFHs $(\operatorname{SFR}(t) \propto$ $\exp (-t / \tau)$ with timescale $\tau=[0.1, \infty]$ and age $t=[0.1,20] \mathrm{Gyr}$ constrained to be shorter than a Hubble time at each redshift). This parametrisation may be inappropriate for actively starforming galaxies (especially at high redshifts, Renzini 2009), because it assumes that all galaxies are observed while having their minimum SFR. However, for the redshift range explored $(z<1)$ and for passively evolving galaxies this assumption does not appreciably affect the mass determinations. We checked, for example, that the inclusion of delayed exponential SFHs $\left(\operatorname{SFR}(t) \propto t / \tau^{2} \times \exp (-t / \tau)\right.$ do not significantly affect the stellar masses estimates. As we noted previously, small secondary bursts may cause a systematic increase in the mass estimate (Fontana et al. 2004). For this study, we adopted the Calzetti et al. (2000) extinction law, solar metallicity (see also Table 1 in Pozzetti et al. 2007), Bruzual \& Charlot (2003) population synthesis models, and a Chabrier IMF (Chabrier 2003) with lower and upper cutoffs of 0.1 and $100 M_{\odot}$, to which we collectively refer hereafter as the default parameters, if not specified otherwise. All GSMFs were computed by using in addition M05 and CB07 population synthesis models. In particular, we are aware that the estimate of ETG stellar masses at high- $z$ are particularly sensitive to the TP-AGB phase of the stellar population around 1-2 Gyr, producing a shift to lower masses of up to 0.2 dex (Maraston et al. 2006). In the redshift range sampled here $(0.1<z<1.0)$, ETGs have on average relatively old stellar populations (Thomas et al. 2005) that are not dominated by the TP-AGB phase, in particular for massive objects. At higher redshifts $(z>1-1.5)$, the effect of the TP-AGB phase in younger (1-2 Gyr) ETGs becomes very important and should not be neglected. In our data (limited to $z<1$ ), we find that by using M05 models instead our main results and conclusions remain almost unchanged and we discuss the differences later in the text.

\section{Galaxy classification}

We used 5 different methods to classify each galaxy as either early- or late-type (ETGs and LTGs, hereafter), according to its colour, star formation activity, morphology, or spectroscopy, as follows:

1. Photometric classification (red and blue galaxies): we derived galaxy photometric types $(\mathcal{P} \mathcal{T})$ from the best-fit to the multi-band photometry (from $u^{*}$ to $K_{\mathrm{s}}$ band, see previous section). Following Zucca et al. (2006), we used the empirical set of (62) SEDs described in Ilbert et al. (2006). These SEDs were derived by interpolating between the four local observed spectra of Coleman et al. (1980, ranging from that of the old stellar population of both M31 and M81 to Sbc, Scd, and Im SEDs) and two starburst SEDs from Kinney et al. (1996). These templates were also linearly extrapolated to the ultraviolet $(\lambda<2000 \AA)$ and near-infrared wavelengths using the GISSEL synthetic models (Bruzual \& Charlot 2003). Galaxies were divided into four types, according to their spectral energy distribution between the UV and near-IR. These types correspond to a red E/Sa template $(\mathcal{P T}=1)$, an early spiral template $(\mathcal{P T}=2)$, a late spiral template $(\mathcal{P T}=3)$, and an irregular or a starburst template $(\mathcal{P T}=4)$. Using the data from the VVDS spectroscopic
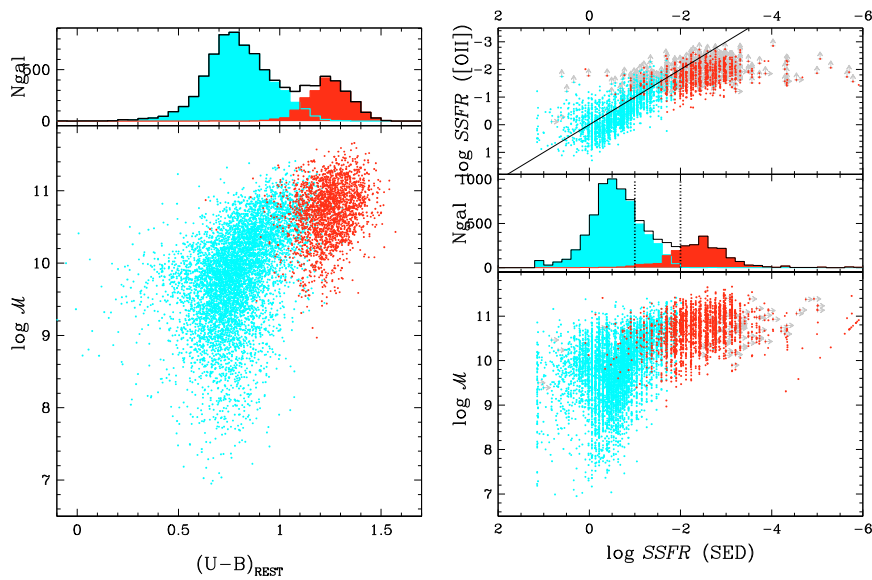

Fig. 1. Left figure: the upper panel shows the $U-B$ rest-frame colour distribution of galaxies with different photometric types. The bottom panel shows the colour-mass diagram. Right figure: SSFR of galaxies with different photometric types. The upper panel shows the comparison between estimates from the SED fitting and from [OII] for galaxies in the range $0.5<z<0.9$. The middle panel shows the SSFR distribution of galaxies, along with the two dotted lines used to separate active from quiescient $\left(\log \left(S S F R / \mathrm{Gyr}^{-1}\right)=-1\right)$ or from passive galaxies $\left(\log \left(S S F R / \mathrm{Gyr}^{-1}\right)=-2\right)$. The bottom panel shows the $\log \left(S S F R / \mathrm{Gyr}^{-1}\right)$ mass diagram. Red dark and light cyan colours represent SED-ETGs $(\mathscr{P T}=1)$ and SED-LTGs $(\mathcal{P T}=2-4)$, respectively. (A color version of this figure is available in the online version of the article.)

survey, Zucca et al. (2006) verified statistically the consistency between the photometric classification and the average galaxy spectral properties. Using stacked spectra of all galaxies of each of the four types, they detected an increasingly bluer continuum with stronger emission lines from $\mathcal{P} \mathcal{T}=1$ to $\mathcal{P} \mathcal{T}=4$, confirming the robustness of this classification scheme.

In Fig. 1 (left panel), we present the $U-B$ rest-frame colour distributions of galaxies in our sample divided according to the photometric types. From this figure, it is evident that the colour bimodality present in the sample can be described well by dividing the sample according to their photometric type: red galaxies that have $\mathcal{P T}=1$ (2103 objects, also called SED-ETGs hereafter) and blue galaxies that have $\mathcal{P T}=$ 2-4 (6347 objects, SED-LTGs), respectively. In Fig. 1 (left panel), we also show the colour-mass diagram, which confirms that when our photometric classification is applied to the 10k-bright zCOSMOS sample it is consistent with previous selections of red-sequence galaxies based on colourluminosity diagrams (Bell et al. 2004; Cimatti et al. 2006) and has the advantage of using the entire multiband photometric coverage rather than only two bands. In principle, given the good multiband coverage, the SED fitting is able to break the degeneracy between a dust-extinguished starforming galaxy with a red but smooth SED and a passive, old galaxy with a strong $4000 \AA$ break in its SED (Pozzetti \& Mannucci 2000).

2. Star formation activity classification (active, quiescent, and passive galaxies): we divided the sample according to the galaxy star formation rate $(S F R)$ activity. We used the $S F R$, and also the specific star formation rate $(S S F R=S F R / \mathcal{M})$ derived from the SED fitting (see previous section) finding, in general, a good correlation between these and the SSFR derived from [OII] (Maier et al. 2009) for galaxies in the redshift range $0.5<z<0.9$ (shown in Fig. 1, right upper panel). 
We therefore divided the sample into active and quiescent galaxies depending on whether $\log \left(S S F R / \mathrm{Gyr}^{-1}\right)$ is above or below -1 , i.e., galaxies that would take less or more than 10 Gyr to double their $\mathcal{M}$ at the present $S F R$, respectively. In our sample, we find 5051 active galaxies and 3403 quiescent galaxies. With this definition, we note that the number of galaxies in the high SSFR sample is $\sim 80 \%$ of the blue galaxies defined on the basis of the SED fitting (see Fig. 1, right middle panel). In addition, we investigate in this paper the population of "passive" galaxies, i.e., 1612 galaxies with $\log \left(S S F R / \mathrm{Gyr}^{-1}\right)<-2$. This dividing value separates well the two parts of the bimodal distribution of the SSFR (Fig. 1, right middle panel).

3. Morphological classification (spheroidal and disc + irregular galaxies): taking advantage of the COSMOS HST+ACS images (Koekemoer et al. 2007), we also divided the sample according to morphological class. To take into account uncertainties in the morphological classification, we used two of the available estimates, i.e., the Zurich Estimator of Structural Types (ZEST; Scarlata et al. 2007a) and a nonparametric estimate derived by our collaborators in Marseille (MRS hereafter, Cassata et al. 2007, 2008; Tasca et al. 2009). Only a small fraction $(\sim 3-4 \%)$ of objects do not have a morphological classification.

ZEST quantitatively describes the galaxy structure by performing a principal component analysis (PCA) in the fivedimensional parameter space of asymmetry (A), concentration (C), Gini coefficient $(\mathrm{G})$, the second-order moment of the brightest 20\% galaxy pixels (M20; Abraham et al. 2003), and the ellipticity of the light distribution $(\epsilon)$. The PCA indicates that the first three PC variables account for more than $90 \%$ of the variance in the original data set, thus almost completely describing the galaxy structure. The ZEST classification associates each PC value with a type (ZEST type, $\mathcal{Z} \mathcal{T}$, =1 for early-type galaxies; =2 for disc galaxies; and $=3$ for irregular galaxies) and a "bulgeness" parameter according to the median value of the distribution of Sérsic indices $n$ (Sargent et al. 2007) of all galaxies brighter than $I=22.5$ (bulgeness $=0-3$, from $n>2.5$ being bulgedominated to $n<0.75$ being disc-dominated galaxies). In this study, the total sample was divided into morpho-ETGs and morpho-LTGs, with the morpho-ETGs sample including 1680 galaxies classified by ZEST as elliptical $(\mathcal{Z} \mathcal{T}=1,759)$ or bulge-dominated $(\mathcal{Z} \mathcal{T}=2.0)$ galaxies, while the morphoLTGs subsample includes 6413 galaxies (with $\mathcal{Z} \mathcal{T}$ greater than 2.0, i.e., from not-bulge-dominated to disc-dominated and irregular galaxies). In addition, we distinguish ellipticals $(\mathcal{Z T}=1)$ from bulge-dominated galaxies $(\mathcal{Z} \mathcal{T}=2.0)$.

For comparison, we also used MRS morphological estimates. This classification scheme separates galaxies on the basis of their position in the multi-dimensional parameter space of the four non-parametric diagnostics of galaxy structures $(\mathrm{C}, \mathrm{A}, \mathrm{G}$, and axial ratio). First, the structural parameters are measured for all galaxies in the sample. Then, a randomly extracted subset of 500 galaxies is visually classified (LT, PC) as ellipticals, spirals, or irregulars. This reference catalogue is used to explore the multi-dimensional parameter space. For each new galaxy that needs to be classified, the distance in the parameter space to the 500 reference galaxies is measured, and the 11 closest selected. Finally, each galaxy is assigned to the most frequent visual class among these 11 nearest reference galaxies. This procedure allows us to convert structural parameters into morphological classes: spheroidals (MRS type, $\mathcal{M T}=1$ ), spirals

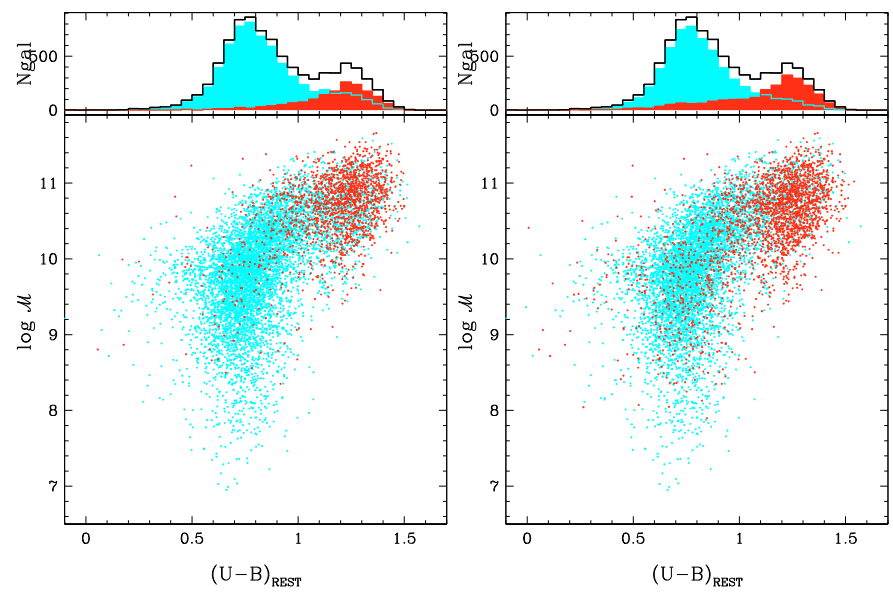

Fig. 2. $U-B$ rest-frame colours of galaxies with different morphological classes using ZEST (left panel) and MRS (right panel) classifications. The colour-mass diagrams are shown in the bottom panels: red dark and light cyan colours represent morpho-ETGs (spheroids) and morpho-LTGs (disc+irregulars), respectively. (A color version of this figure is available in the online version of the article.)

$(\mathcal{M T}=2)$, and irregulars $(\mathcal{M T}=3)$. For this paper, we also therefore used this morphological classification to divide the sample into morpho-ETGs and morpho-LTGs, as in the case of ZEST morphologies, yielding 2383 spheroids $(\mathcal{M T}=1)$ and 5831 disc+irregular $(\mathcal{M T}=2+3)$ galaxies, respectively. In Fig. 2, we show the $U-B$ rest-frame colour distributions and colour-mass diagrams of these different morphological classifications. For both morphological classifications, there is a non-negligible number of blue spheroidal-type galaxies ( $\sim 37 \%$ in MRS and $\sim 29 \%$ in ZEST) (see Sect. 8.2) as well as red disc+irregular galaxies $(\sim 13 \%$ in ZEST and $\sim 9 \%$ in MRS).

4. Spectroscopic classification (emission- and absorptionline galaxies): using measurements of spectral features (Lamareille et al., in prep.), we divided the sample into spectral-ETGs and spectral-LTGs following the criteria defined in Mignoli et al. (2009) for the zCOSMOS spectroscopic sample in the $E W_{0}[\mathrm{OII}]-D 4000$ plane. In the redshift range $0.55<z<1.0$, we find 1079 galaxies without strong emission lines and large $4000 \AA$ breaks $\left(E W_{0}([\mathrm{OII}])<5 \AA\right.$ and $D 4000+0.33 \log \left(E W_{0}([\mathrm{OII}])\right)>$ 1.5 , and spectroscopic type $\mathcal{S T}=1$, i.e., spectral-ETGs) and 3304 galaxies with strong emission lines and small $4000 \AA$ breaks $\left(E W_{0}([\mathrm{OII}])>5 \AA\right.$ and $1.50<D 4000+$ $0.33 \log \left(E W_{0}([\mathrm{OII}])\right)<2.22$, spectroscopic type $\mathcal{S} \mathcal{T}=2$, i.e., spectral-LTGs), respectively.

5. Combined classification: a clean sample of "bona fide ETGs": given the remaining controversy about morphology or colour-selected early-type galaxies (see Franzetti et al. 2007 , for a discussion of the contamination colour-selected samples), we defined a more conservative sample of "bona fide ETGs" by combining different criteria related to colours, morphology, and spectral properties. From the sample of red galaxies (with photometric type $=1$ ), we selected only those whose properties are more accurately described by the 4 reddest templates $(\sim 70 \%)$ and we removed galaxies with either strong emission lines $\left(E W_{0}([\mathrm{OII}])\right.$ or $E W_{0}\left(\mathrm{H}_{\alpha}\right)>$ $5 \AA, \sim 30 \%$ ) or reliably classified disc+irregular morphologies (using the intersection between MRS and ZEST estimates, $\sim 20 \%)$ or strong emission at $24 \mu \mathrm{m}(K-m(24 \mu \mathrm{m})>$ $-0.5, \sim 5 \%)$, obtaining a final sample of 981 red passive 
spheroids ("bona fide ETGs"). For further details, we refer to Moresco et al. (2010).

\section{The galaxy stellar mass function}

\subsection{The method}

To derive the galaxy stellar mass function $(G S M F)$, we follow traditional techniques used in computing the luminosity function. Here we apply the classical non-parametric $1 / V_{\max }$ formalism (Schmidt 1968) and estimate the best-fit Schechter (1976) parameters $\left(\alpha, \mathcal{M}^{*}, \phi^{*}\right)$. In performing the fit, we allow ourselves to use up to 2 Schechter function components.

To correct for both the non-targeted sources in spectroscopy and those for which the spectroscopic redshift measurement failed, we use a statistical weight associated with each galaxy with a secure redshift measurement. This weight is the inverse of the product of the target sampling rate (TSR) and the spectroscopic success rate (SSR). Accurate weights were derived by Bolzonella et al. (2009; see also Zucca et al. 2009) for all objects with secure spectroscopic redshifts, taking into account the magnitude, colour, and redshift dependence of the SSR, as well as the objects observed as compulsory $(\sim 2 \%)$ and secondary objects $(\sim 2 \%)$ in the slits, which have different TSRs from the whole sample.

\subsection{The limits in mass}

In a magnitude-limited sample, the minimum stellar mass for which observations were completed depends on both the redshift and the stellar mass-to-light ratio $\mathcal{M} / L$. This latter quantity obviously depends on the stellar populations and therefore on galaxy colours. To account for this limit, we define at each redshift a minimum mass, $\mathcal{M}_{\min }$, above which the derived GSMF is essentially complete because all types of galaxies are potentially observable above this mass.

To derive $\mathcal{M}_{\min }$, we calculate the limiting stellar mass $\left(\mathcal{M}_{\text {lim }}\right)$ of each galaxy, i.e., the mass it would have, at its spectroscopic redshift, if its apparent magnitude were equal to the limiting magnitude of the survey $\left(I_{\text {lim }}=22.5\right)$ given by $\log \left(\mathcal{M}_{\text {lim }}\right)=$ $\log (\mathcal{M})+0.4\left(I-I_{\lim }\right)$. The result is a distribution of limiting stellar masses, $\mathcal{M}_{\mathrm{lim}}$, that reflects the distribution of stellar $\mathcal{M} / L$ ratios at each redshift in our sample. To derive a representative limit for our sample, we use the $\mathcal{M}_{\text {lim }}$ of the $20 \%$ faintest galaxies at each redshift. This choice takes into account the colourluminosity relation and therefore includes only galaxies with a typical $\mathcal{M} / L$ close to the magnitude limit. By doing this, we avoid the artificial use of a too stringent limit related to the brightest and reddest (with the highest $\mathcal{M} / L$ ) galaxies, which do not significantly contribute close to the magnitude limit of the survey. Figure 3 shows the distribution of stellar masses for all galaxies and of $\mathcal{M}_{\mathrm{lim}}$ for the $20 \%$ faintest galaxies. We then define $\mathcal{M}_{\min }(z)$ as the upper envelope of the $\mathcal{M}_{\text {lim }}$ distribution below which lie $95 \%$ of the $\mathcal{M}_{\lim }$ values at each redshift. This $\mathcal{M}_{\min }$ corresponds to a $95 \%$ completeness limit to the $\mathcal{M} / L$ ratio at each redshift observable by the survey, and is taken to be the completeness limit of the GSMF. Meneux et al. (2009) instead used mock survey samples to derive a mass limit. For a given redshift range and mass threshold, the completeness was simply defined as the ratio of the number of galaxies brighter than the observed flux limit to those of all fluxes. Interestingly, even if this method is model-dependent, it leads to a similar completeness limit as the previous one. For example, for the global population we find that our $\mathcal{M}_{\min }$ are in fairly good agreement

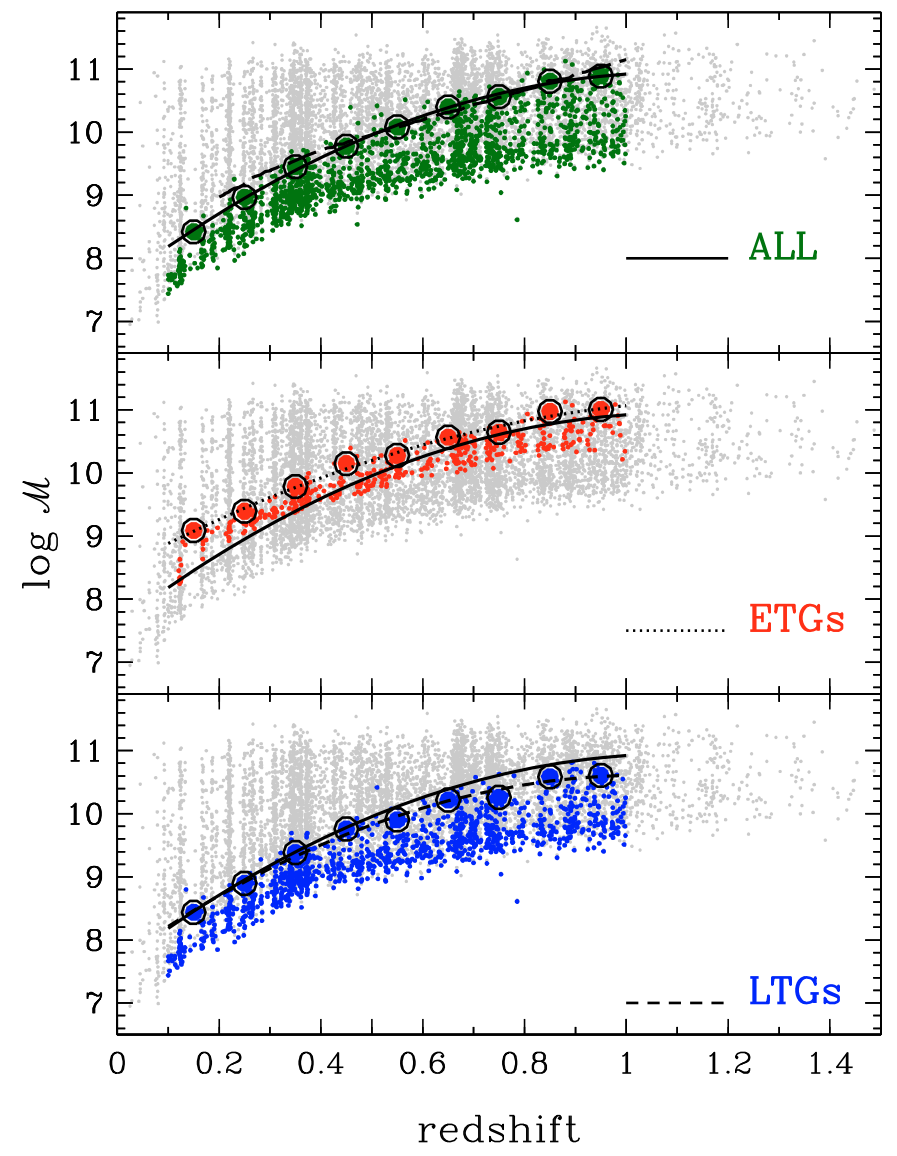

Fig. 3. Stellar mass as a function of redshift (small grey dots). Also shown are $\mathcal{M}_{\text {lim }}$ (intermediate size dark dots) and $\mathcal{M}_{\text {min }}$ (big black circles and lines) at $95 \%$ of the $\mathcal{M} / L$ completeness level (see text): upper panel for the total population, middle and lower panel for SEDETGs and SED-LTGs, respectively, defined by their photometric type. Also shown with a dashed black line is the $\mathcal{M}$ threshold (corresponding to $95 \%$ of the completeness level for the global GSMF) derived using mock Millenium samples by Meneux et al. (2009).

with the 95\% completeness limit (black curve in Fig. 3) derived by Meneux et al. (2009) for zCOSMOS, in most of the redshift range, and in any case the completeness for $\mathcal{M}>\mathcal{M}_{\text {min }}$ is never lower than $85 \%$ at any redshift.

We derived $\mathcal{M}_{\min }$ at each redshift and for each galaxy subsample used. For the global population, we note that $\mathcal{M}_{\text {min }}$ at low redshift is close to the limit of the bluest population (see Fig. 3), because blue galaxies dominate the zCOSMOS population close to the magnitude limit at that redshift, while the global $\mathcal{M}_{\text {min }}$ shifts towards that of the reddest population at high redshift (at which redshift a significant number of red galaxies is present at the limit of our survey, and have a higher $\mathcal{M} / L$ and therefore higher mass limit).

The $G S M F$ s derived using the $1 / V_{\max }$ technique, which corrects in addition for volume incompleteness, are formally complete for $\mathcal{M} \geq \mathcal{M}_{\min }\left(z_{\text {inf }}\right)$, i.e., $\mathcal{M}_{\text {min }}$ at the lowest redshift of the considered bin. In the parametric fit, we estimate the best-fit Schechter parameters using data above $\mathcal{M}_{\text {min }}\left(z_{\text {inf }}\right)$ and plotting as lower limits the data below $\mathcal{M}_{\text {min }}$, where the GSMFs are incomplete. In addition, we took into account the upper limits that are above the maximum mass found in each considered redshift bin, deriving $1 \sigma$ upper limits by following Gehrels (1986) in the case of Poisson statistic for $n=0$ events (i.e., $\leq 1.84$ at $1 \sigma$ ). 


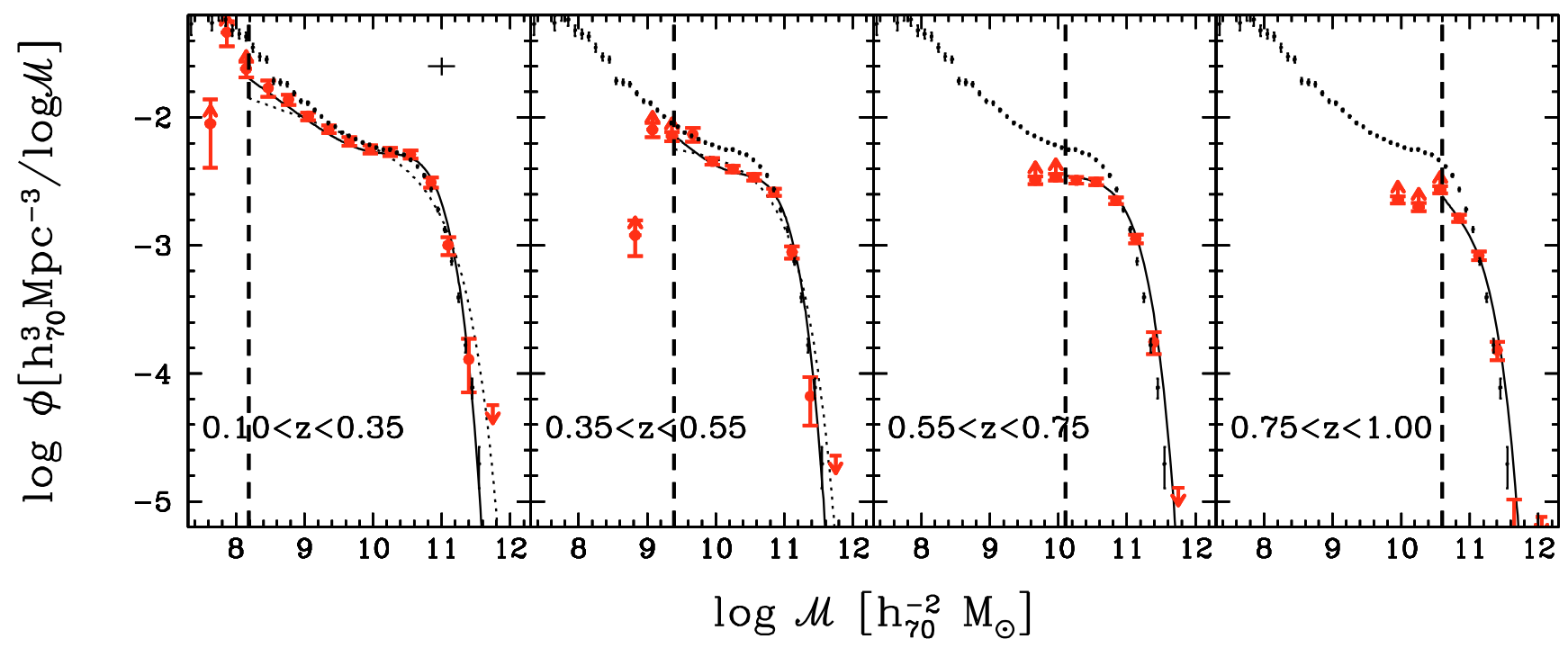

Fig. 4. Global galaxy stellar mass functions in four different redshift bins. Red filled points represent $1 / V_{\max }$ determination and associated Poisson errors, while the lines represent the Schechter fits. The global GSMFs are not reproduced well by a single Schechter function (dotted lines), but by two Schechter functions (continuous lines) up to $z=0.55$. In the first two redshift bins, the GSMFs show the inflection points of the bimodality around $\mathcal{M} \sim 5 \times 10^{9} \mathcal{M}_{\odot}$. The dashed vertical lines represent the mass limit in the corresponding redshift bin $\left(\mathcal{M}_{\min }\right)$. Data are plotted as lower limits below $\mathcal{M}_{\min }$. Upper limits at $1 \sigma$ (i.e. 1.84 objects) are shown at the high-mass end. Small black dots in all panels represent the local GSMF by Baldry et al. (2008). The cross at the right top of the first panel shows an estimate of the cosmic variance and mass uncertainties.

\section{The bimodality in the zCOSMOS global galaxy stellar mass function}

The resulting GSMFs of the zCOSMOS galaxy sample were derived for the redshift range $0.1<z<1$. We divided this redshift range into 4 redshift bins to obtain an approximately comparable number of objects in each redshift bin. This choice also dilutes the effect of the most prominent large-scale structures at all redshifts (Lilly et al. 2009; Kovač et al. 2010b). Using the deviations from the median number densities in numerous redshift bins, affected by different structures, we estimate that the systematic error in our GSMFs caused by cosmic variance is about $15-20 \%$.

Figure 4 shows the global GSMFs in different redshift bins derived using the $1 / V_{\max }$ technique, with the associated Poisson errors. The most clearly evident results are that at $z<0.55$ the global GSMFs, derived using the $1 / V_{\text {max }}$ technique, are bimodal, exhibit an upturn with a steep slope below $\mathcal{M} \sim 10^{9.5} \mathcal{M}_{\odot}$, and are not reproduced well by a single Schechter function. At higher redshifts, the narrower mass range due to the increase in $\mathcal{M}_{\text {min }}$ does not allow us to explore the upturn in the low-tointermediate mass regime. We therefore allowed the fit to include up to two Schechter functions. We verified that the characteristic mass $\left(\mathcal{M}^{*}\right)$ of the second Schechter function, which dominates at low mass, is poorly constrained and consistent with the characteristic mass of the Schechter function dominating at high mass. Therefore, following Baldry et al. (2008), we considered hereafter a double Schechter function with a single $\mathcal{M}^{*}$ value, given by

$\phi(\mathcal{M}) \mathrm{d} \mathcal{M}=\mathrm{e}^{-\mathcal{M} / \mathcal{M}^{*}}\left[\phi_{1}^{*}\left(\frac{\mathcal{M}}{\mathcal{M}^{*}}\right)^{\alpha_{1}}+\phi_{2}^{*}\left(\frac{\mathcal{M}}{\mathcal{M}^{*}}\right)^{\alpha_{2}}\right] \frac{\mathrm{d} \mathcal{M}}{\mathcal{M}^{*}}$,

where $\phi_{\mathcal{M}} \mathrm{d} \mathcal{M}$ is the number density of galaxies with mass between $\mathcal{M}$ and $\mathcal{M}+\mathrm{d} \mathcal{M}$, and $\alpha_{2}<\alpha_{1}$, so that the second term dominates at the lowest masses.

Using galaxies with $\mathcal{M} \geq \mathcal{M}_{\min }$, we find that the slope parameters of the 2 Schechter functions differ significantly. Using the F-test, we find that the fit with two Schechter functions is

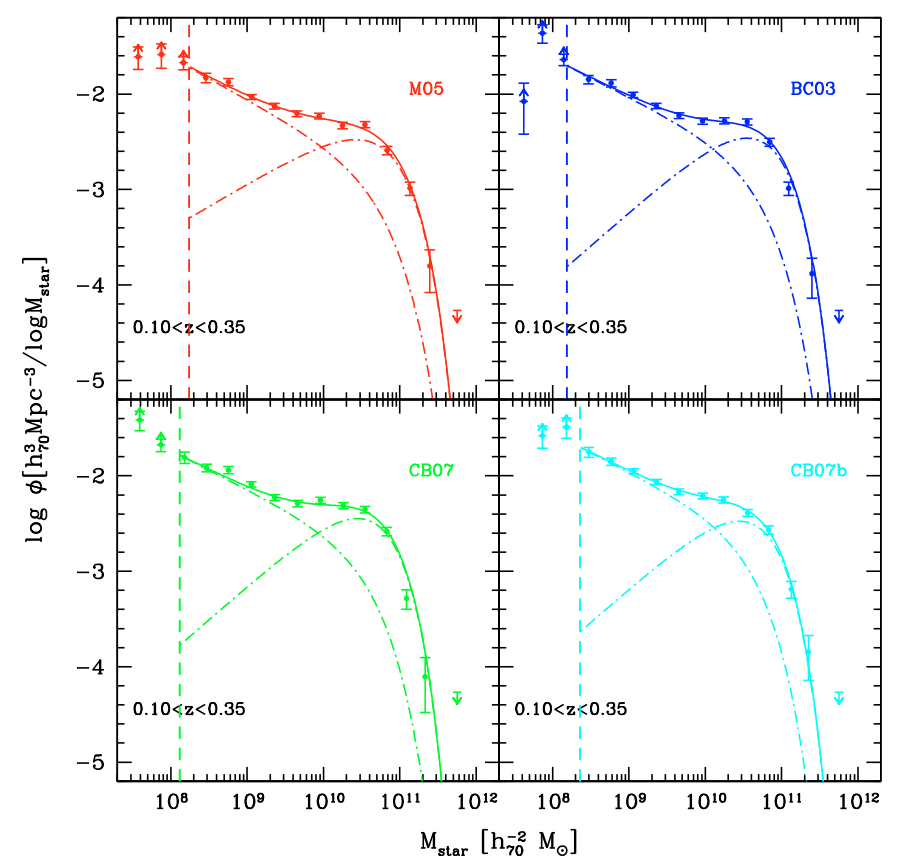

Fig. 5. Global GSMF in the first redshift bin using different SFHs and population synthesis models to derive the stellar masses (BC03, M05, $\mathrm{CB} 07, \mathrm{CB} 07 \mathrm{~b}=\mathrm{CB} 07+$ secondary burst). Points and lines have the same meaning as in Fig. 4. The dot-dashed lines represent the 2 Schechter components: they intersect around $\mathcal{M} \simeq 10^{10} \mathcal{M}_{\odot}$. (A color version of this figure is available in the online version of the article.)

tighter than the fit with a single function at $\geq 3 \sigma$ confidence level in the first two redshift bins. We checked that our findings for the double Schechter shape are insensitive to the particular SFHs and set of population synthesis models used to estimate $\mathcal{M}$. This is shown in Fig. 5, where we plot the GSMF derived in the first redshift bin $(0.1<z<0.35)$ using different SFHs and population synthesis models to obtain the mass estimates. 
The strength of the bimodality in the global GSMF is related to the mutual ratio of the two Schechter functions that dominate the fit at the high and low-mass end, respectively. In Sect. 8, we explore whether the populations of ETGs and LTGs can explain this bimodality in the GSMF.

For many years, there has been evidence of a "faint-end upturn" in the optical galaxy luminosity functions, whereby the luminosity function is found to rise steeply about 3-5 mag below the characteristic luminosity $\left(L^{*}\right)$, in both clusters (Driver et al. 1994; Popesso et al. 2006) and the field (Zucca et al. 1997; Blanton et al. 2005). We note, however, that the upturn is not evident for all samples (Norberg et al. 2002). The existence of a similar upturn is less clearly evident in the GSMF. Baldry et al. (2004, 2006, 2008) fitted a similar shape to the local GSMF in the SDSS with an upturn at masses below $\sim 10^{9} \mathcal{M}_{\odot}$. Baldry et al. (2008) converted the galaxy luminosity function of rich galaxy clusters in the SDSS (Popesso et al. 2006) to an equivalent mass function and found an even more prominent upturn in their GSMFs than that found for the field GSMF. This upturn has not been clearly investigated until now in the GSMFs derived from deep surveys, even if a substantial population of low-mass galaxies $\left(<10^{9} M_{\odot}\right)$ at low redshift $(z \simeq 0.2)$, consisting of faint blue galaxies, has been noticed in deep surveys such as the VVDS (Pozzetti et al. 2007). We note, a posteriori, by comparing with other GSMFs from deep surveys (VVDS, Deep2, MUSIC, GOODS, FDF, COMBO17) that previous surveys (in particular MUSIC, Fontana et al. 2006; and COMBO17, Borch et al. 2006) show some hint of bimodality in the shape of their GSMFs, even up to $z \sim 1$ (see Fig. 10 in Pozzetti et al. 2007). Ilbert et al. (2010) also found that the GSMFs derived in COSMOS using photometric redshifts cannot be reproduced well with a single Schechter function, but the upturn is less evident than in the zCOSMOS sample. This may be caused by the higher precision of the $\mathcal{M}$ determination achieved by using spectroscopic redshifts instead of photometric ones.

In the zCOSMOS survey, the dependence of the GSMF shape, on the galaxy environment, as seen in the local Universe (Baldry et al. 2006), is investigated in a parallel paper (Bolzonella et al. 2009), in which the bimodality in the global mass function is found to be even stronger in high density enviroments.

In the following sections, we study the evolution of the global GSMF and how its shape is related to the bimodality in the galaxy properties. We derive the contribution of the different galaxy types to the GSMF and its bimodal shape, and we explore its evolution with cosmic time. In Sect. 7.1, we also explore how the evolution related to SFHs, which differ between high- and low-mass galaxy populations, changes the shape of the GSMF bimodality.

\section{The evolution of the galaxy stellar mass function}

We explore how the evolution of the global GSMF with cosmic time depends on galaxy mass. From Fig. 4, a mass-dependent evolution of the global GSMFs is clearly evident: the massive tail is almost stable up to $z=1$, while the number density of less massive galaxies increases continuously with cosmic time, in agreement with previous studies (Fontana et al. 2006; Pozzetti et al. 2007). This mass-dependent evolution suggests that most of the massive galaxies assembled their mass earlier than lower mass galaxies ("mass-assembly downsizing"). Figure 6 shows the number densities $\left(\rho_{N}\right)$, derived by the $1 / V_{\max }$ method, for two different mass limits of $\log \left(\mathcal{M} / \mathcal{M}_{\odot}\right)>9.77,10.77$ (corresponding to $\log \left(\mathcal{M} / \mathcal{M}_{\odot}\right)>10,11$ for a Salpeter (1955) IMF, as often

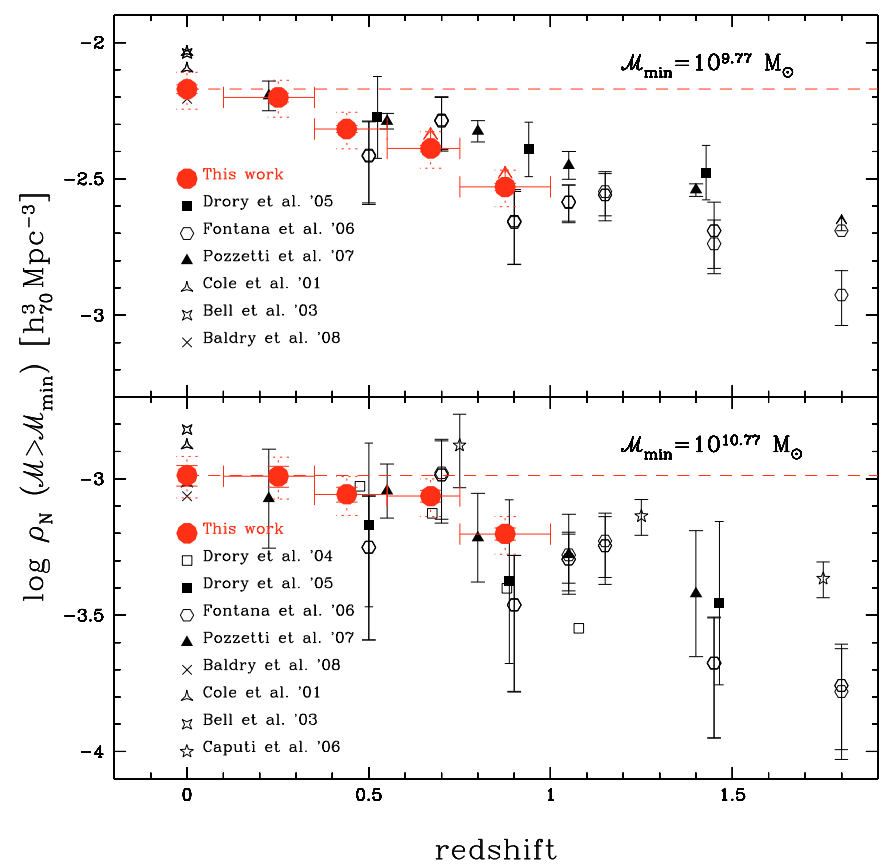

Fig. 6. Cosmological evolution of the galaxy number density as a function of redshift, derived using $1 / V_{\max }$ from the zCOSMOS for 2 different mass thresholds $\left(>10^{9.77} M_{\odot}\right.$ and $>10^{10.77} M_{\odot}$ from top to bottom). Dotted errors include cosmic variance estimates. zCOSMOS data at $z=0$ have been derived from the "SFH-evolved GSMFs" (see Sect. 7 and Fig. 8). The dashed lines correspond to the no-evolution solution normalized at $z=0$. Results from previous surveys (small black points) are also shown. (A color version of this figure is available in the online version of the article.)

used in the literature). We compare our results with literature data from other high- $z$ deep surveys (VVDS, DEEP2, GOODSMUSIC, FORS Deep Field, COMBO17; see Pozzetti et al. 2007, for details and references), and for the local Universe (Cole et al. 2001; Bell et al. 2003; Baldry et al. 2008). The local values for zCOSMOS were derived assuming an evolution from the lowest redshift bin to $z=0$ (see next section for details). zCOSMOS data are consistent with most previous observations at $z<1$ and are more accurately determined. We confirm a continuous evolution in number density for $\log \left(\mathcal{M} / \mathcal{M}_{\odot}\right)>9.77$, which increases towards that of the local Universe, and a slightly milder evolution for $\log \left(\mathcal{M} / \mathcal{M}_{\odot}\right)>10.77$, which is negligible for $z<0.7$ $(<20 \%)$ and more rapid above this redshift $(<60 \%$ since $z \sim 1)$.

The same trend with mass is evident in Fig. 7, which shows the number densities $\left(\rho_{N}\right)$ as a function of redshift using a more extended range in mass limits (from $\log \left(\mathcal{M} / \mathcal{M}_{\odot}\right)>9$ to $\left.\log \left(\mathcal{M} / \mathcal{M}_{\odot}\right)>11.3\right)$. Over the entire mass range, our data are consistent with a faster and steeper increase in the number densities with cosmic time going from high to low-mass galaxies: the evolution from $z=0.44$ to $z=0$ is $0.12 \pm 0.02 \mathrm{dex}$ for $\log \left(\mathcal{M} / \mathcal{M}_{\odot}\right)>9.5$ and $0.09 \pm 0.03$ for $\log \left(\mathcal{M} / \mathcal{M}_{\odot}\right)>10.6$. Only galaxies with $\log \left(\mathcal{M} / \mathcal{M}_{\odot}\right)>11$ show negligible evolution from $z=1$ to the present time $(0.03 \pm 0.07 \mathrm{dex})$, while the evolution is $0.19 \pm 0.03 \mathrm{dex}$ for $\log \left(\mathcal{M} / \mathcal{M}_{\odot}\right)>10.6$, the lowest mass for which we are complete over the whole redshift range.

zCOSMOS data are consistent with a mass-dependent assembly history, with more massive galaxies evolving earlier than lower mass galaxies (mass-assembly downsizing). We therefore confirm that mass is an important parameter driving galaxy evolution. In the context of the hierarchical scenario, galaxy formation is predicted to be controlled primarily by their dark 


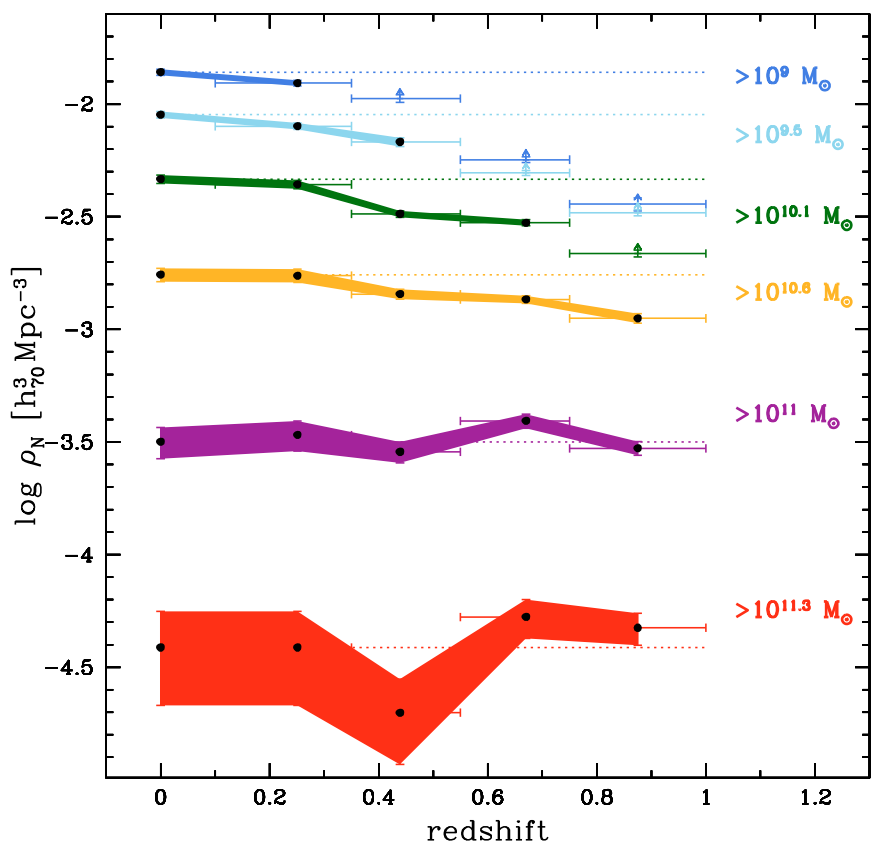

Fig. 7. Number density evolution using different mass limits for stellar masses. zCOSMOS data at $z=0$ have been derived from the "SFH-evolved GSMFs" (see Sect. 7 and Fig. 8). The dotted lines correspond to the no-evolution solution normalized at $z=0$. (A color version of this figure is available in the online version of the article.)

matter halo mass, and therefore, one would expect the evolution of individual galaxies to be affected also by their enviroment. The relative importance of stellar mass and galaxy environment to the shape and evolution of the GSMF is investigated for the zCOSMOS dataset in a parallel paper (Bolzonella et al. 2009). This study found an accelerated trend of downsizing in overdense regions (see also Iovino et al. 2010).

A detailed comparison of the global GSMF with hierachical semi-analytical models (SAM) is postponed to a future paper. In Sect. 9, we compare the predictions of SAMs with observational ETG GSMFs. Here we recall that most hierarchical galaxy assembly models are unable to fully account for the observed GSMF and its evolution (see Fontana et al. 2004, 2006; Caputi et al. 2006; Kitzbichler \& White 2007; Marchesini et al. 2009; Fontanot et al. 2009, for a detailed comparison with models). A revision of the physical treatment of the baryonic component, such as its star-formation history/timescale, of the role of feedback, dust content, and/or AGN feedback (Menci et al. 2006; Monaco et al. 2007; Bower et al. 2006), and the introduction of the uncertainties in the mass determination, may help to reduce the disagreement (Kitzbichler \& White 2007; Cattaneo et al. 2008; Fontanot et al. 2009). Fontanot et al. (2009) compare a broad compilation of available data sets with the predictions of three different semi-analytic models of galaxy formation within the $\Lambda C D M$ framework. When observational errors on stellar mass are taken into account, they also find that the models acceptably reproduce the observed number density of massive galaxies $\left(\log \left(\mathcal{M} / \mathcal{M}_{\odot}\right)>11\right)$, but that low-mass galaxies $\left(\log \left(\mathcal{M} / \mathcal{M}_{\odot}\right)=9-10\right)$ are predicted to form too early in the models and are too passive at late times. Thus, the models do not correctly reproduce the downsizing trend in stellar mass. We show in Sect. 9 that the typical uncertainties in the mass determination cannot fully account for the observed excess of massive galaxies relative to some hierarchical model predictions, and, when applied, the hierarchical models tend to overestimate the high-mass galaxy number density at low redshift (see also Fig. 1 in Fontanot et al. 2009).

In the following section, we analyse whether the evolution of the observed GSMF with cosmic time is driven mainly by the SFHs at any given mass, and quantify the residual importance of merging events.

\subsection{The importance of the SFHs to the evolution at $z<1$}

We attempt to interpret the mass-dependent evolution with cosmic time found for the $G S M F$, i.e., the increase in number density at a given $\mathcal{M}$ or the shift in $\mathcal{M}$ at a given number density, in terms of only a "pure growth in stellar mass" with cosmic time related in turn to the mass-dependent star-formation history of each galaxy (Thomas et al. 2005; Noeske et al. 2007a,b). In this way, we aim to quantify the residual importance of merging events or other processes after accounting for the role of the galaxy star formation activity, which drives the growth in stellar mass with cosmic time. A "SFH driven evolution" was also adopted by Bell et al. (2007), assuming a constant specific SFR between two adjacent redshift bins and an instantaneous return fraction of $45 \%$.

For each galaxy, we derived the $\mathcal{M}$ evolved following its SFH (exponentially decreasing) derived from the SED fitting. Indeed, the best-fit parameters of the stellar population models (such as the star-formation history timescale $\tau$ and the age) derived from the observed SEDs, allow us to follow the evolution of stellar mass in cosmic time, based on the assumption that the galaxies evolved in isolation (i.e., without merging) and continued to form stars in a smooth way with the same SFH derived from the SED fitting. For each galaxy at redshift $z$, we derived the $\mathcal{M}$ evolved to the mean redshift of the previous (i.e., lower) redshift bin. The cosmic time elapsed between the mean redshifts of two adjacent redshift bins ranges between 1.1 and 1.6 Gyr. For galaxies in the lowest redshift bin $\left(z_{\text {mean }}=0.25\right)$, we predicted the evolved $\mathcal{M}$ to $z=0$, i.e., after about $3 \mathrm{Gyr}$. We performed simulations to test the reliability of this "evolved stellar mass" $\left(\mathcal{M}_{\text {evolved }}\right)$ : using models, we created a simulated multi-band catalogue at the depth of our catalogue (following Bolzonella et al. 2000), from which we were able to recover not only the present $\mathcal{M}$ of the input model (see also Pozzetti et al. 2007) but also the model "evolved stellar mass" after a given time; the agreement overall is satisfactory, with no systematic shifts and with the typical dispersions caused by statistical uncertainties and degeneracies being of the order of $0.13-0.21 \mathrm{dex}$, depending on the elapsed time.

We find that the mass growth derived from the SED fitting in our sample is on average about a factor $1.7(0.24 \mathrm{dex})$ for $\mathcal{M}=10^{9.5} \mathcal{M}_{\odot}$ and $1.22(0.09 \mathrm{dex})$ for $\mathcal{M}=10^{10} \mathcal{M}_{\odot}$, but reaches a factor of 30 for the most extreme star-forming galaxies with $\log \left(\mathcal{M} / \mathcal{M}_{\odot}\right)<10$. In Fig. 8, we show the GSMF predicted at the average redshift of each bin using the galaxies in the higher redshift bin and compare it with the measured GSMF at the observed redshift. In the first panel, we also show the GSMF predicted at $z=0$ using galaxies in the first redshift bin $(0.1<z<0.35)$.

First of all, we note a modest evolution from $z \simeq 0.25$ to $z=0$, in particular for low-to-intermediate mass galaxies $\left(\log \left(\mathcal{M} / \mathcal{M}_{\odot}\right)<10\right)$ with a shift towards higher masses being related to the SFHs. The evolved GSMF approaches that observed locally (Baldry et al. 2008) with which it is rather consistent for $\log \left(\mathcal{M} / \mathcal{M}_{\odot}\right)>9$.

For the zCOSMOS data set, we find reasonable agreement at each redshift between the "SFH-evolved GSMF" and that 


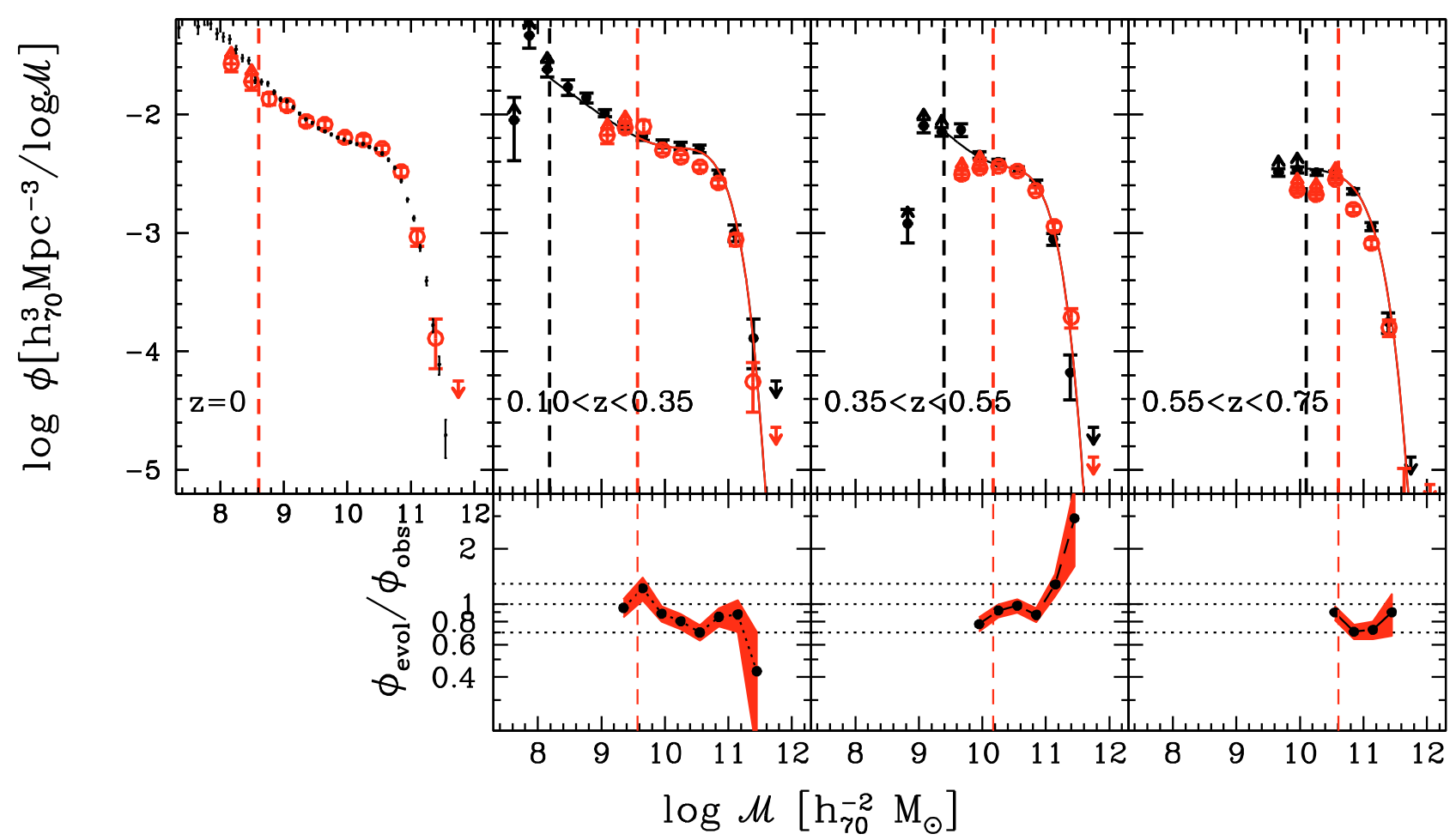

Fig. 8. Upper panels: "SFH-evolved GSMF" (empty red points) compared to observed GSMF at redshift $z$ (black filled points, from Fig. 4). In the first panel, we plot the $G S M F$ evolved from the first redshift bin to $z=0$ (empty red points), compared with the local GSMF (Baldry et al. 2008, shown as small dots). Vertical lines represent the respective mass limits. Lower panels: ratio of "SFH-evolved GSMF" to observed GSMF (both estimated using $1 / V_{\max }$ technique). The dotted lines correspond to $\pm 30 \%$ around 1 .

observed at the same redshift. Close agreement at $z>0.3$ was also found by Bell et al. (2007) for COMBO-17 by assuming a constant SFH. Our result suggests that the GSMF evolution is, therefore, driven mostly by smooth and decreasing SFHs, because of the progressive exhaustion of the gas reservoir, for example from cold gas accretion, rather than by merger events or major bursts. Very similar results of a large contribution to the mass growth rate by the less massive and star-forming galaxies were obtained independently by Vergani et al. (2008) in the VVDS. Figure 8 (lower panel) shows the ratio of the evolved to the observed GSMFs in the first three redshift bins for $\mathcal{M}>\mathcal{M}_{\min }$ of evolved galaxies. The agreement is good particularly of intermediate-to-low masses $\left(\log \left(\mathcal{M} / \mathcal{M}_{\odot}\right)<10.6\right)$, where most of the GSMF evolution occurs and could therefore be explained mainly by the growth in mass driven by the SFHs in intermediate-to-low mass galaxies. At higher masses, galaxies have lower SSFRs and therefore the SFHs are unable to explain the evolution observed in the MF, which in any case is quite small (see Fig. 7). In the next section, we explore the role of mergers in the evolution of the GSMF.

At all masses, we find differences at most of $\sim 40 \%$, and even lower than $20 \%$ for $\log \left(\mathcal{M} / \mathcal{M}_{\odot}\right)<10.3$, between the evolved and observed GSMFs. These residual differences may be related to different processes. Among them, one possibility is an additional SFR, i.e., either more prolonged SFHs than inferred from the SED fitting, a different SFH functional form (Renzini 2009), or secondary bursts. From the SED fitting, we find that for the blue galaxies the timescale $(\tau)$ distribution for the exponential SFHs has a median value of $1 \mathrm{Gyr}$, even if extended to higher values. This median timescale is quite short relative to the value inferred from the global SFHs (the compilation of Hopkins \& Beacom 2006, is consistent with $\tau \sim 3.5 \mathrm{Gyr}$ at $z<1$ ). On the other hand, the residual differences could also be explained by merger events (see next section) or other dynamical processes that are able to induce a growth in stellar mass unrelated to the SFH.

Here, we also checked that the predicted colour distributions for the "SFH-evolved galaxies" were consistent with the observed colour distributions at different redshifts. Figure 9 compares the expected colour distributions to the observed ones at different redshifts for two extreme hypotheses about the dust content: (A) (left panel): no evolution in the dust content, i.e., assuming the same dust content at all redshifts as derived from the SED fitting at the observed redshift, or (B) (right panel) no dust content in the evolved galaxies. In both panels, we compare the colour distribution for galaxies in a mass-limited sample using the most conservative limit $\mathcal{M}_{\text {min }}\left(z_{\text {sup }}\right)$ for evolved masses. Model A predicts too red colours at all redshifts, while only the extreme case of model B, i.e., without any dust content, is consistent with the observed colour distributions, at least at $z>0.35$, while, in any case, it predicts too red colours at lower redshift. This could be related (again) to a too rapid decline in decreasing (quenching) of the SFRs of each galaxy as inferred by the SED fitting technique. We therefore conclude that more extended SFHs or secondary bursts appear to be necessary to more accurately reproduce the observed colours, in particular at low-redshift. One possibility would be to directly model the observed decrease/quenching of the $S F R$ at later cosmic times for progressively lower masses (see also Sect. 4). We postpone the discussion of this possibility to a future paper.

\subsection{A limit to the galaxy merging}

The small amount of evolution observed in the GSMF argues against a dominant contribution of galaxy mergers to galaxy evolution. If merger events, indeed, were efficient in forming the 
L. Pozzetti et al.: zCOSMOS: Galaxy bimodality in the stellar mass function

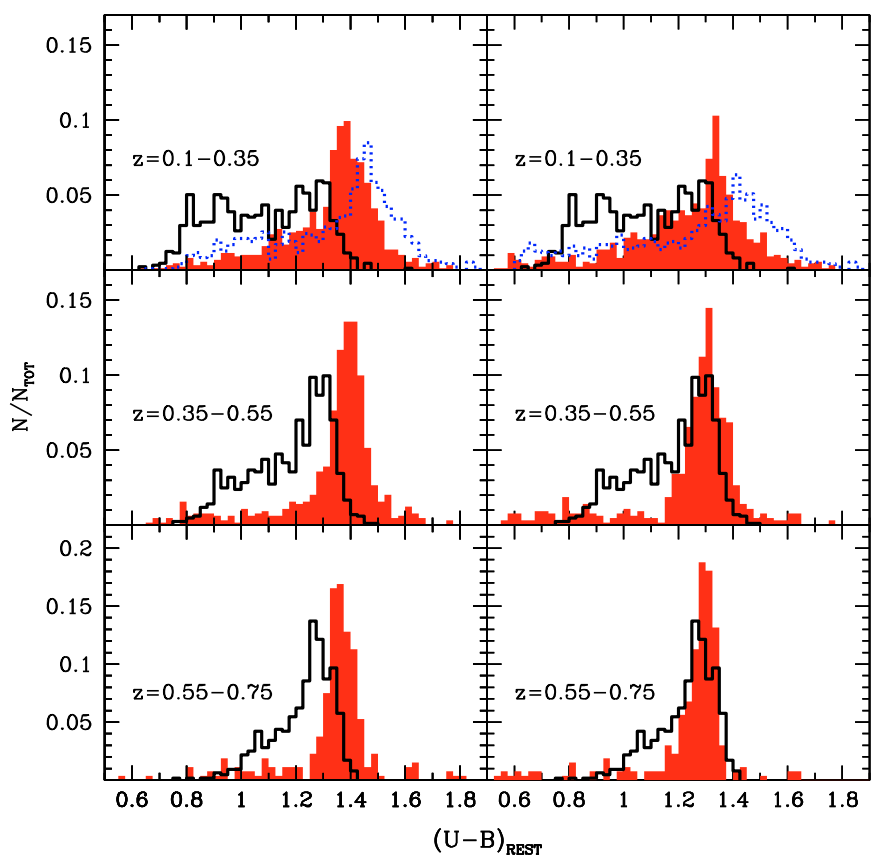

Fig. 9. Rest-frame $U-B$ colour distribution in a mass-limited sample (using the most conservative limit, see text) in different redshift bins, as observed (solid black histogram for the total population) and predicted using the "SFH-evolution" from the previous higher redshift bin (red filled histogram). The dotted blue histogram in the top panels represent the expected $z=0$ colour distribution by applying the "SFH-evolution" to the galaxies in the first redshift bin. Left panels: assuming no evolution in the dust content, i.e., the same dust content derived from the SED fitting at the observed redshift; right panels: assuming no dust content in the evolved galaxies.

most massive galaxies at $z<1$ (as predicted by hierarchical models), we should observe instead a detectable increase in the number density of massive galaxies. In particular, the negligible evolution in the $G S M F$ for galaxies with $\log \left(\mathcal{M} / \mathcal{M}_{\odot}\right)>11$ up to $z \simeq 1$ suggests that these galaxies formed and assembled their stellar mass at higher redshifts, ruling out a major role for mergers at $z<1$.

We derived the contribution of mergers to the evolution of the GSMF. We first estimated the growth rate of the number densities for different $\mathcal{M}$, after accounting for the growth in mass produced by ongoing star formation (see previous section). The residual growth rate of the number density was calculated to be

$\dot{\rho}_{N}(\mathcal{M}, z)=\frac{\left[\rho_{N}^{\mathrm{obs}}(\mathcal{M}, z)-\rho_{N}^{\mathrm{evol}}\left(\mathcal{M}, z_{\text {sup }}\right)\right]}{\Delta t\left(z, z_{\text {sup }}\right)}$,

where $\rho_{N}$ was evaluated using the $1 / V_{\max }$ method. The result is shown in Fig. 10 (panel a) as a function of $\mathcal{M}$ in the various redshift bins. We compare these values with the major-merger rate $\left(\dot{\rho}_{\mathrm{mm}}\right)$ derived from galaxy pairs by de Ravel et al. (2010) for the zCOSMOS sample. It is evident that major-merger rate is always below the growth rate by a factor of 2 or more, except in the intermediate redshift range $(z \sim 0.5)$. This suggests that major mergers do not contribute significantly to the residual evolution. We, therefore, derived the merger rate (major+minor) that could explain the residual evolution. We estimated the effective increase in number densities at any given $\mathcal{M}$ by accounting also for galaxies that at the same time leave the current mass bin due to major merging. This estimate represents the number of galaxies produced by mergers (major+minor) per Gyr per $\mathrm{Mpc}^{3}$ to explain the GSMF growth at that mass. Finally, assuming that

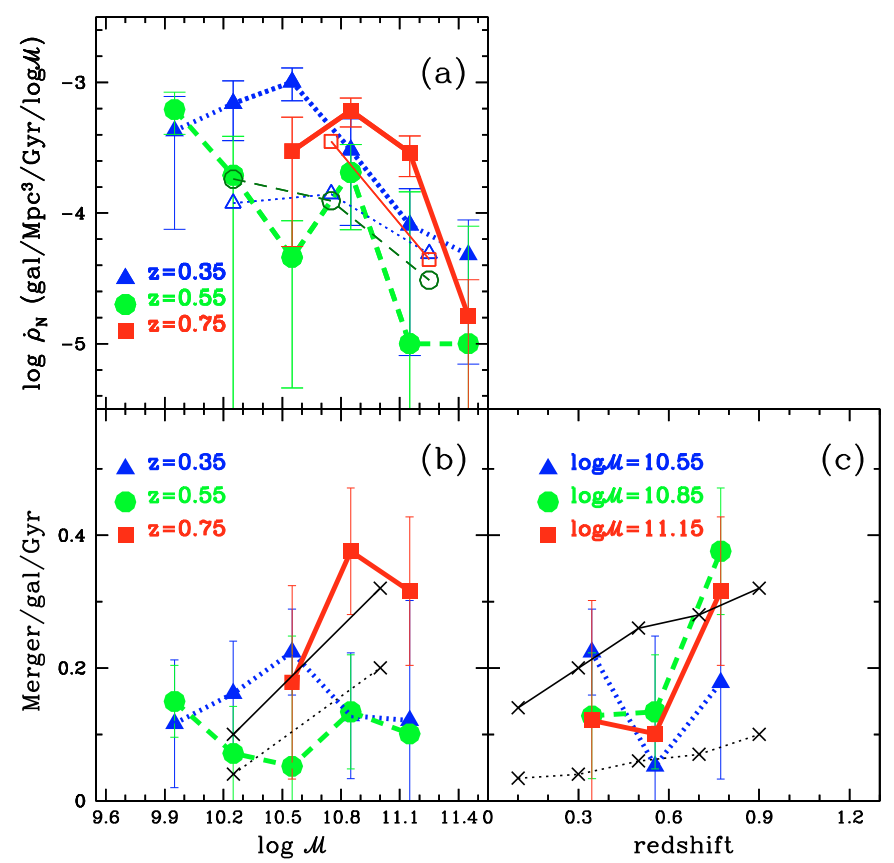

Fig. 10. Panel a): growth rate in number density after accounting for star formation evolution. Different thick lines and filled colour points represent estimates at different redshifts (dotted, dashed and solid for $z \sim 0.35,0.55$, and 0.75 , respectively). For comparison, thin lines and empty points are the major merger rate estimates by de Ravel et al. (2010). Panel b): merger per Gyr per galaxy as a function of mass. Same lines and points as in panel a). Predictions based on cosmological simulations from Stewart et al. (2009), for mergers with mass ratio $m / \mathcal{M}>0.1$, are plotted as thin lines and black cross for the lowest and highest redshift bin. Panel c): merger per Gyr per galaxy as a function of redshift. Thick lines and points represent various mass ranges (dotted, dashed and solid for $\log \left(\mathcal{M} / \mathcal{M}_{\odot}\right) \sim 10.55,10.85,11.15$, respectively). Predictions from Stewart et al. (2009) for $m / \mathcal{M}>0.1$ are plotted as thin lines and black cross for $\log \left(\mathcal{M} / \mathcal{M}_{\odot}\right) \sim 10.3$ (dotted line) and $\log \left(\mathcal{M} / \mathcal{M}_{\odot}\right)>11$ (solid line)

a galaxy is the product of the last single merger (within galaxy pairs of any mass ratio $m / \mathcal{M}$ ), we derived the number of mergers per galaxy per Gyr dividing by the number densities of galaxies. We note, indeed, that the time interval between two adjacent redshift bins is not long $(1-1.5 \mathrm{Gyr})$ relative to the typical merger timescale used in SAMs (De Lucia et al. 2010) or predicted by numerical simulations (Boylan-Kolchin et al. 2008) that prevent multiple minor mergers predominating. We, therefore, derive the merging rate per galaxy per $\operatorname{Gyr}\left(f_{\mathrm{M}}\right)$ in the following way:

$f_{\mathrm{M}}(\mathcal{M})=\frac{\left[\dot{\rho}_{N}(\mathcal{M}, z)+\dot{\rho}_{\mathrm{mm}}\left(\mathcal{M}, z_{\text {sup }}\right)\right]}{\rho_{N}(\mathcal{M}, z)}$ merger $/$ gal $/ \mathrm{Gyr}$

The results are shown in Fig. 10 as a function of mass (panel b) and redshift (panel c). At high redshift, we find for massive galaxies $\left(\log \left(\mathcal{M} / \mathcal{M}_{\odot}\right)>10.6\right.$ at $\left.z>0.5\right)$ that the merger rate is quite high, $0.2-0.4$ merger $\mathrm{Gyr}^{-1} \mathrm{gal}^{-1}$, but decreases rapidly to $\sim 0.1$ with cosmic time, being only marginally consistent with an increase with mass. At lower redshifts, the merger rate does not show any clear dependence on mass and always remains below 0.2 at all masses. On average, we measure 0.16 merger $\mathrm{Gyr}^{-1} \mathrm{gal}^{-1}$ since $z=1$. The major merger rate per galaxy derived by De Ravel et al. (2010) is always below 0.1 at all redshifts and masses explored ( $\sim 0.06$ on average). Integrated over cosmic time (from $z \simeq 1$ to $z \simeq 0.1$ ), massive galaxies $\left(\log \left(\mathcal{M} / \mathcal{M}_{\odot}\right) \sim 10.6\right)$ have experienced about 0.7 merger $\mathrm{gal}^{-1}$ 

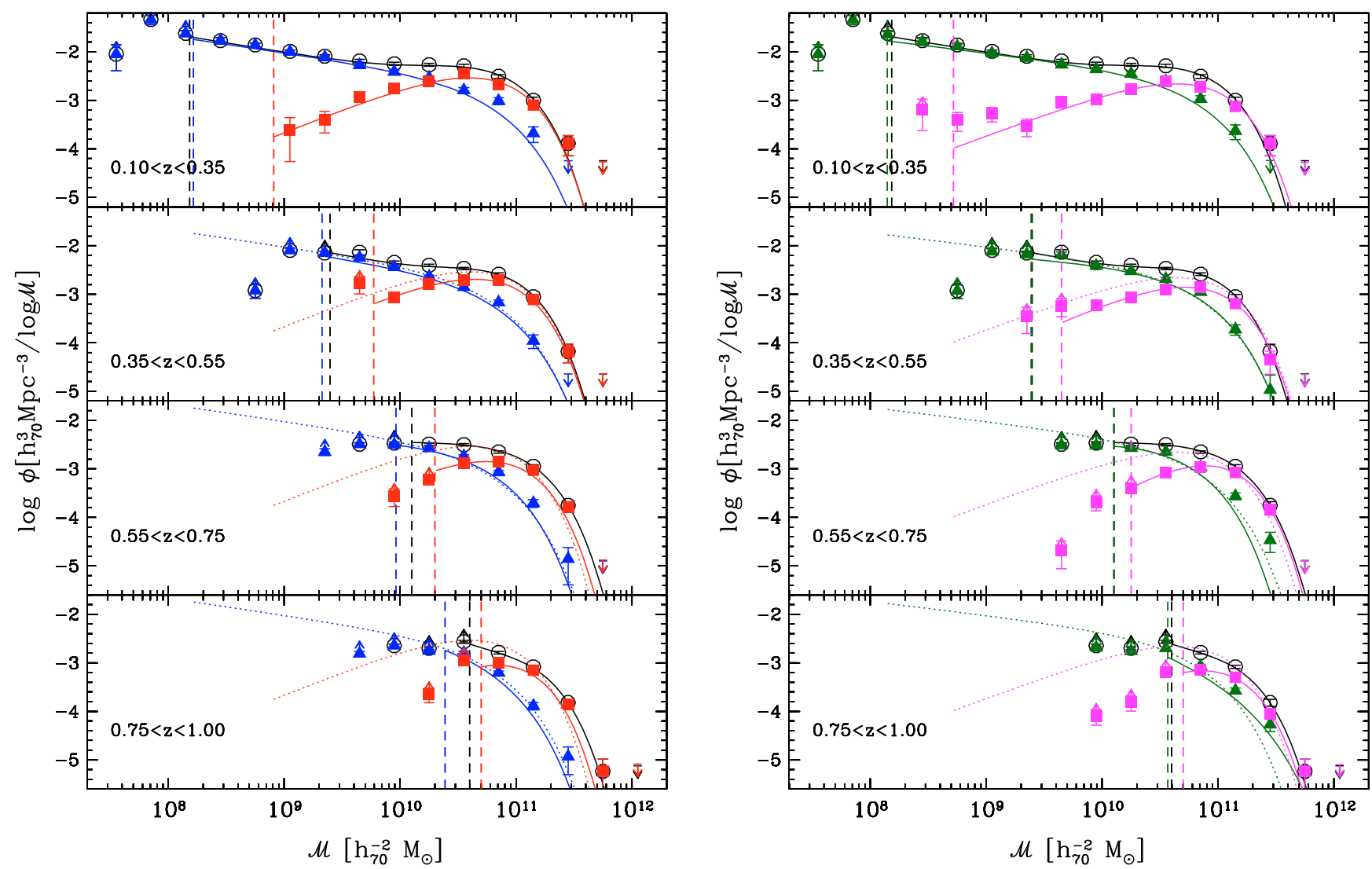

Fig. 11. Galaxy stellar mass function by galaxy types. Left panel: GSMFs by photometric types $(\mathcal{P T}=1$ as red squares, $\mathcal{P} \mathcal{T}=2+3+4$ as blue triangles, total population as empty black circles). Right panel: GSMFs for morphological types $(\mathcal{Z T}=1,2.0$ as magenta squares and $\mathcal{Z} \mathcal{T}>2.0$ as dark green triangles). In both panels, points represent the $1 / V_{\max }$ determination, while continuous lines the Schechter fits. Dotted lines reported in each panel, as a reference, are the Schechter fits to the first redshift bin. Dashed vertical lines represent the mass limit for the corresponding redshift bin $\left(\mathcal{M}_{\min }\right)$. (A color version of this figure is available in the online version of the article.)

$(<0.3$ below $z \sim 0.7)$, of which less than 0.2 are major mergers. In Fig. 10 we compared our estimates with the model predictions of Stewart et al. (2009) for mergers within galaxy pairs of mass ratio greater than $0.1(m / \mathcal{M}>0.1)$. Galaxy mergers in a hierarchical $\Lambda \mathrm{CDM}$ scenario increase with stellar mass, redshift, and decrease with $m / \mathcal{M}$ ratio. Minor mergers contribute considerably more than major mergers, dominating in all but the most massive galaxies (Parry et al. 2009). Indeed, even in hierarchical formation models, most of ellipticals and spiral bulges acquire their stellar mass through minor mergers or disc instabilities. Given our uncertainties, we cannot draw any firm conclusions about either the redshift or mass dependences, even if our data appear to exclude a strong dependence on mass, at odds with hierarchical predictions. There is, instead, some evidence of an increase with redshift, qualitatively in agreement with models.

We therefore conclude that the role of major merging events is not dominant and cannot completely explain the evolution of the GSMF, even after accounting for the growth in mass produced by ongoing star formation. Mergers in general (major+minor) at a rate of between 0.1 and $0.4 \mathrm{Gyr}^{-1} \mathrm{gal}^{-1}$ may account for the residual evolution, these rates possibly decreasing with cosmic time but not having a clear dependence on mass. Oesch et al. (2010) discuss in greater detail the role of merging in the evolution of massive galaxies within the COSMOS survey. In a future paper on the final zCOSMOS sample, we will explore in detail the role of both major and minor merging on the evolution of the GSMF.

\section{Mass functions by galaxy types}

To explore the bimodality observed in the GSMF and how it depends on both time and galaxy-type evolution, we derived a typedependent GSMF using the different classifications described in Sect. 4.

Figure 11 (left panel) shows the GSMF divided into different photometric types, red $(\mathcal{P T}=1)$ versus blue $(\mathcal{P T}=2-4)$ galaxies; the GSMFs obtained by using morphological criteria to differentiate between ETGs and LTGs are shown in the right panel of the same figure. We find that the red/spheroidal and blue/disc+irr populations have very different GSMFs, which can be linked to the bimodality of the global GSMF. The GSMFs of both ETGs and LTGs are reproduced quite well by a single Schechter function. The LTG GSMFs exhibit a steep low-mass end, which in constrast with is flat for the ETGs at all redshifts. However, we find a hint of a small upturn in both the GSMFs of ETGs for the morpho-ETGs (see Fig. 11) and in the second redshift bin of our other ETG classifications (see Fig. 16). Drory et al. (2009), instead, found that neither their red (passive) nor their blue (star-forming) galaxy stellar mass functions could be fitted well with a single Schechter function, but show an upturn at low masses. In general, for all the criteria adopted, we find that the massive end of the mass function $\left(\mathcal{M}>10^{10.5} \mathcal{M}_{\odot}\right)$ is mainly dominated by ETGs (red/spheroidal/passive) up to $z=1$, while LTGs (blue/disc+irr/active) mostly contribute to the intermediate/low-mass part $\left(\mathcal{M}<\mathcal{M}^{*}\right)$ of the mass function at 


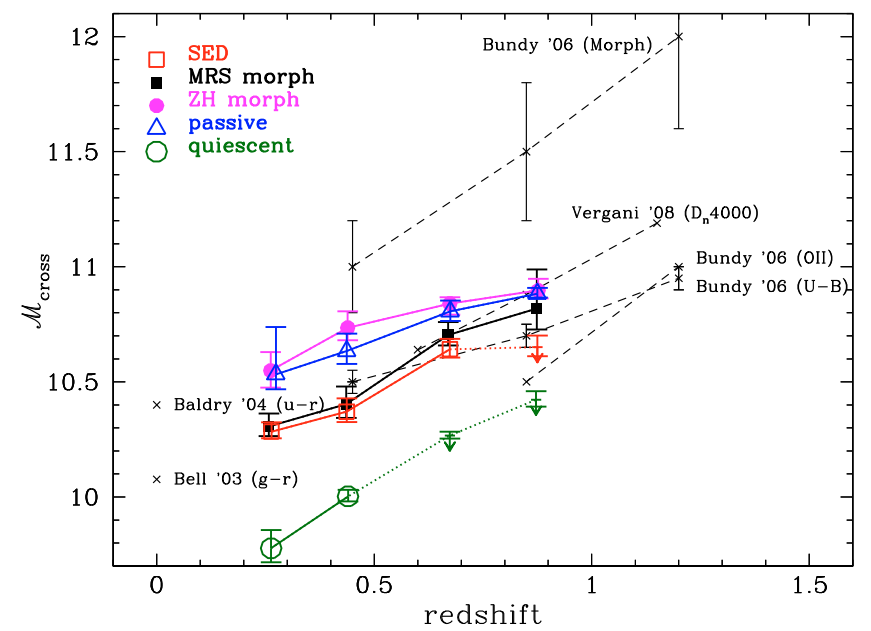

Fig. 12. The intersection of the GSMFs $\left(\mathcal{M}_{\text {cross }}\right)$ of the two populations (ETGs and LTGs) as a function of redshift. Values were derived from the $1 / V_{\max }$ estimate of the GSMFs. Different symbols (and colours) refer to the different classification schemes adopted (see figure legend), upper limits are shown where $\mathcal{M}_{\text {cross }}$ is lower than $\mathcal{M}_{\text {min }}(z)$. Black crosses show data from the literature (references are marked, along with the respective classification criteria).

all redshifts. For $\log \left(\mathcal{M} / \mathcal{M}_{\odot}\right)>11$, the number density of blue galaxies is always below $\sim 2 \times 10^{-4} \mathrm{gal} / \mathrm{Mpc}^{3} / \log \mathcal{M}$.

Using the $1 / V_{\max }$ data points of the GSMFs of ETGs and LTGs, we derived the intersection of the two populations $\left(\mathcal{M}_{\text {cross }}\right)$. For the photometric classification, $\mathcal{M}_{\text {cross }}$ is at $\sim 1.9( \pm 0.2) \times 10^{10} \mathcal{M}_{\odot}$ in the first redshift bin and evolves with redshift, increasing by about a factor of 2 (see red circles in Fig. 12) by $z=1$. Although for somewhat higher values of $\mathcal{M}_{\text {cross }}$, a similar trend with redshift was found using the morphological classifications to divide the sample into spheroids and disc+irregular galaxies, for both classification schemes explored in this paper. We determined similar values of $\mathcal{M}_{\text {cross }}$ by dividing the sample in terms of the star formation activity (active versus passive with $\log \left(S S F R / \mathrm{Gyr}^{-1}\right)=-2$ ), while $\mathcal{M}_{\text {cross }}$ is quite low when quiescient galaxies are considered $\left(\log \left(S S F R / \mathrm{Gyr}^{-1}\right)=-1\right)$. Our results are consistent with previous determinations of $\mathcal{M}_{\text {cross }}$ in other deep surveys, such as VVDS (Vergani et al. 2008) and DEEP2 (Bundy et al. 2006), the only exception being the value of $\mathcal{M}_{\text {cross }}$ derived by Bundy et al. (2006) using morphological types (the highest line in Fig. 12). In this case, the difference may be caused by a different and more extreme definition of morphological ETGs by Bundy et al. (2006). The extrapolation of our $\mathcal{M}_{\text {cross }}$ to $z=0$ is also consistent with local results (Baldry et al. 2004; Bell et al. 2003).

The evolution of $\mathcal{M}_{\text {cross }}$ is indicative of an increase/decrease in the fraction of early/late galaxies with cosmic time at intermediate masses, as already noted in previous surveys (Fontana et al. 2004; Bundy et al. 2006; Arnouts et al. 2007; Scarlata et al. 2007b; Vergani et al. 2008). Oesch et al. (2010) found similar results within the COSMOS survey using extremely robust photometric redshifts. Bolzonella et al. (2009) found a more rapid evolution of $\mathcal{M}_{\text {cross }}$ in high density environments (see also Iovino et al. 2010; and Kovač et al. 2010a, for groups). However, previous surveys have not clearly established the precise evolution in absolute number densities of the two populations, in particular for late-type galaxies. By comparing the zCOSMOS GSMFs for different redshift bins, we find that the evolution of $\mathcal{M}_{\text {cross }}$ is caused mainly by a clear increase with cosmic time in the number density of red/spheroids of intermediate mass (around $\mathcal{M}^{*}$

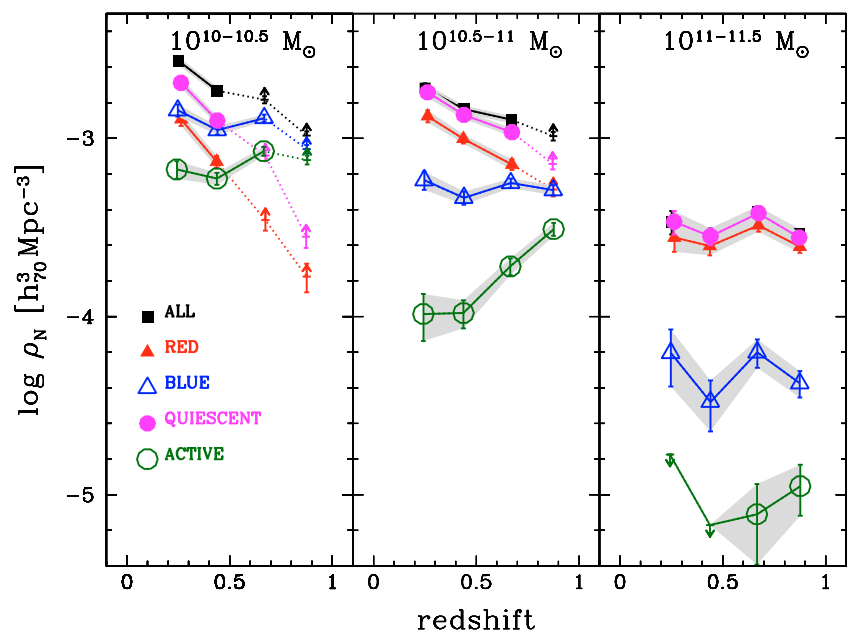

Fig. 13. Number density evolution in different mass ranges. Different symbols and colours refer to different population classes: black squares refers to the total population, empty red and filled blue triangles to SED-ETGs and SED-LTGs using photometric classification, while filled magenta and empty green circles refer to quiescent and active populations, respectively. Hatched regions refer to GSMF complete in the mass range and redshift considered, while dotted lines and lower limits are plotted at redshifts where the GSMF is not complete in the considered mass range. (A color version of this figure is available in the online version of the article.)

and below $10^{11} \mathcal{M}_{\odot}$ ), coupled with only a marginal evolution in the number density of blue/spiral galaxies at the same masses.

These trends become even more distinctive when plotting the number densities, derived using the $1 / V_{\max }$ technique, versus redshift in different mass ranges $\left(\log \left(\mathcal{M} / \mathcal{M}_{\odot}\right)=10-11.5\right)$, as shown in Fig. 13. We find that the number density of massive $\left(\log \left(\mathcal{M} / \mathcal{M}_{\odot}\right)>11\right)$ red types is almost constant up to $z=1$ (see also Pozzetti et al. 2003; Scarlata et al. 2007b; Ilbert et al. 2010) and dominates the total number density at these masses, while the number density of intermediate-mass $\left(10^{10}-10^{11} \mathcal{M}_{\odot}\right)$ red types increases with cosmic time. This increase is steeper at lower masses. Between $z \simeq 0.45$ and $z \simeq 0.25$, it is $0.05 \pm 0.08$, $0.14 \pm 0.04$, and $0.24 \pm 0.05$ dex for the three considered mass ranges of $\log \left(\mathcal{M} / \mathcal{M}_{\odot}\right)=11,10.5$, and 10 , respectively. An independent study (Brown et al. 2008), using halo occupation distributions, shows that while very massive halos often double in mass over the past $7 \mathrm{Gyr}$, the stellar masses of their central galaxies typically grow by only $30 \%$. Our result is indicative of a mass-assembly downsizing as already noted for the global population and even more clearly in the build-up of the red sequence (Cimatti et al. 2006).

In contrast, we find that the number density of late blue types with $\mathcal{M}>10^{10} \mathcal{M}_{\odot}$ remains approximately constant with cosmic time from $z=1$. Their evolution in number density is less than 10-20\% over the entire redshift and mass range (Fig. 14 upper panel). These results are consistent with those of Arnouts et al. (2007), who found that the integrated stellar mass density of the active population shows only a modest mass growth rate, in contrast to an increase by a factor of 2 for the quiescent population. Bundy et al. (2006) found that the abundance of blue galaxies declines by $0.1-0.2$ dex from $z=0.75-1.0$ to $z=0.4-0.7$ at $\log \left(\mathcal{M} / \mathcal{M}_{\odot}\right)=10.6-11.3$ (see their Fig. 6). A small evolution of the "blue cloud GSMF" was also detected by Bell et al. (2007) in the COMBO-17 survey out to $z \simeq 0.9$.

How is this small, possibly negligible evolution in the blue $G S M F$ s related to the global decrease with cosmic time in the 


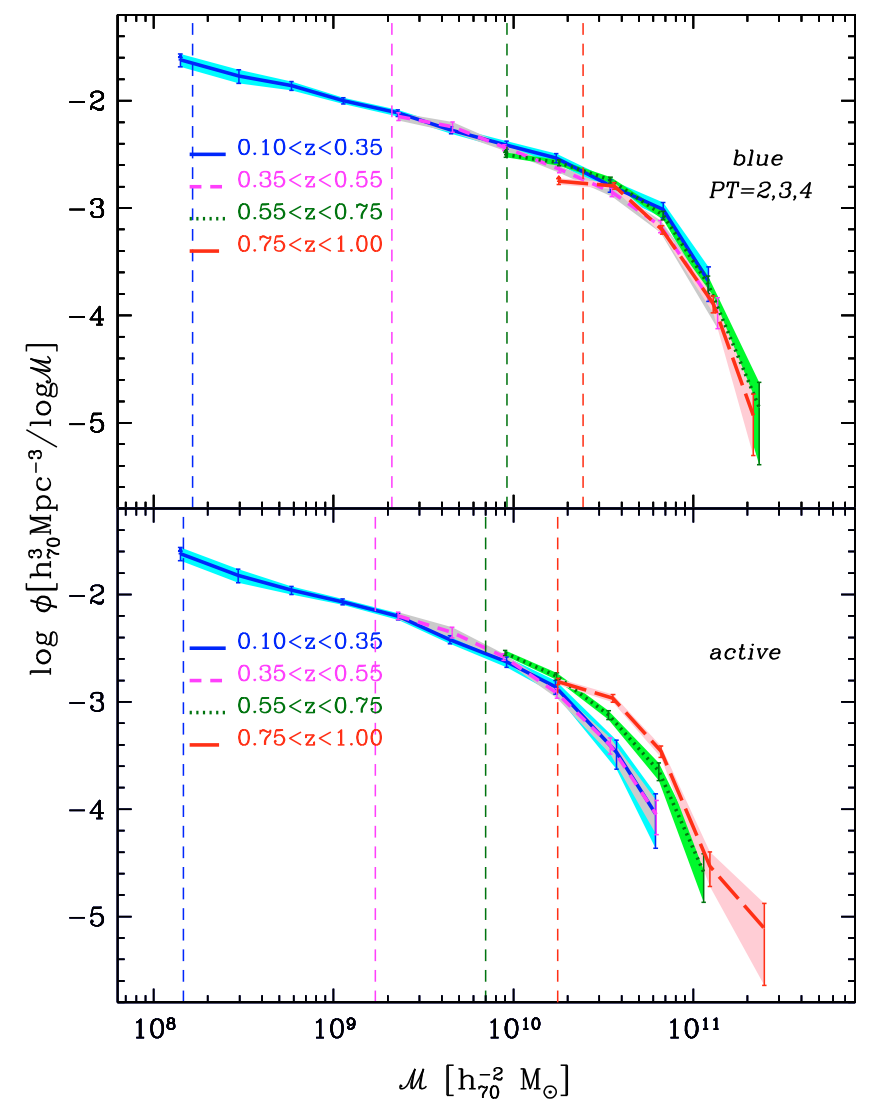

Fig. 14. GSMFs for LTGs using two classification schemes. Upper panel: blue galaxies $(\mathcal{P T}=2,3,4)$, and lower panel: active galaxies $\left(\log \left(\operatorname{SSFR} / \mathrm{Gyr}^{-1}\right)>-1\right)$, as defined in Sect. 4. Only $1 / V_{\max }$ determinations have been plotted, with their uncertainties. Different lines and colours refer to different redshift range (see legend).

SFR density since $z \sim 1$ (see Hopkins et al. 2006) and the downsizing in the SFR (Cowie et al. 1996)? To answer these questions, we analysed the GSMFs of galaxies with different $S F R$ activities. In particular, we derived the GSMFs for the most extreme star-forming population, i.e., those with high $S S F R$.

Using estimates of the $S F R$ and $S S F R$ derived from the [OII] lines (Moustakas et al. 2006, calibration), Maier et al. (2009) find that very few zCOSMOS galaxies with masses above $\log \left(\mathcal{M} / \mathcal{M}_{\odot}\right)>10.8$ have $1 /$ SSFRs below the age of the universe at $0.5<z<0.7$, while there exist several dozens of galaxies above the same mass that are strongly star-forming at $0.7<z<0.9$ (see Fig. 2 in Maier et al. 2009). The same trend is observed using the $S F R$ and SSFR derived from the fit to the multi-band photometry with synthetic models. Using an SED fitting determination, we note, first of all, that the median $S F R$ and $S S F R$ decreases with cosmic time in massive blue galaxies with $\log \left(\mathcal{M} / \mathcal{M}_{\odot}\right)>10.5$ (for example: the median $\log \left(\operatorname{SSFR} / \mathrm{Gyr}^{-1}\right)$ is -1.0 at $0.7<z<1.0$, while it is -1.4 at $z<0.3$ ).

We therefore derived the GSMFs using the SSFR derived from the SED fitting and by dividing the sample as described in Sect. 4, i.e., as those defining active and quiescent galaxies with $\log \left(S S F R / \mathrm{Gyr}^{-1}\right)$ above or below -1 . Figure 14 (lower panel) shows indeed a decrease with cosmic time in the GSMFs of the high-SSFR galaxies, in particular for $\log \left(\mathcal{M} / \mathcal{M}_{\odot}\right)>10.3$. Their GSMF shows, indeed, a mass-dependent evolution that is stronger at the massive end. In the highest redshift bin $(0.75<$ $z<1$ ), the GSMF of the high-SSFR galaxies increases in number density and approaches that previously derived for the sample of blue (SED-LTG) galaxies.

By comparing the GSMFs of the blue galaxies with those of high-SSFR galaxies, we find that, while the number density of blue galaxies overall remains approximately constant, it decreases significantly with cosmic time for high-SSFR massive objects (see Fig. 14). From $z \sim 0.85$ to $z \sim 0.25$, the number density of high-SSFR active galaxies with $\log \left(\mathcal{M} / \mathcal{M}_{\odot}\right)>10.5$ decreases by a factor of $\sim 3(-0.49 \pm 0.12$ dex $)$. Therefore, the decrease with cosmic time in the number of active massive galaxies is balanced by the constancy in intermediate-activity blue galaxies and the increase of intermediate-mass quiescent and red galaxies. Studying the $B$-band luminosity function in zCOSMOS, Zucca et al. (2009) found results consistent with a scenario in which some blue galaxies are transformed into red galaxies with increasing cosmic time.

To summarize, these data suggest that with cosmic time we are witnessing a transformation from active to passive galaxies and a corresponding decrease (increase) in the fraction of late (early) types. These changes in galaxy fractions are caused mainly by the clear increase with cosmic time in the number density of intermediate-mass $\left(\mathcal{M} \sim 10^{10}-10^{11} \mathcal{M}_{\odot}\right)$ early-type galaxies, while the density of intermediate-mass blue or morphoLTGs remains almost constant, being continually replenished by blue active galaxies of even lower masses. The median SSFR of blue massive galaxies decreases with cosmic time. Therefore, blue highly star-forming (of high SSFR) galaxies of intermediate mass $\left(\log \left(\mathcal{M} / \mathcal{M}_{\odot}\right)=10-11\right)$ increase in mass but decrease in either SFR or SSFR with cosmic time, transforming into low$S S F R$ blue objects and, after the quenching of their $S F R$, into red passive objects of intermediate mass. Less-massive, blue, active objects $\left(\log \left(\mathcal{M} / \mathcal{M}_{\odot}\right)<10\right)$ increase in mass to replace in the $G S M F$ s the blue intermediate-mass objects, whose density consequently remains almost constant with time. In agreement with our results, Bell et al. (2007) found that, if there were a growth in stellar mass with a constant SFR equal to the instantaneous one (after subtracting $45 \%$ of the return fraction), the GSMFs of blue galaxies would be dramatically overproduced. By only assuming that all the growth in stellar mass is added to the red sequence, they reproduced the evolution of the blue and red stellar mass functions with remarkable accuracy.

\subsection{The build-up of the red sequence}

We attempt to quantify the growth rate (in terms of both number and mass density) of red galaxies as a function of redshift in different mass ranges. This rate can be interpreted as the flux required to describe the migration of galaxies with cosmic time from the blue cloud to the red sequence.

Figure 15 shows the evolution with redshift for the red population $(\mathcal{P T}=1)$, in different mass ranges, of the growth rate in number density $\left(\dot{\rho}_{N}\right.$, upper panel), estimated using $1 / V_{\max }$ data points. For $\log \left(\mathcal{M} / \mathcal{M}_{\odot}\right)<11$, the growth rate is in the range of $10^{-4}-10^{-3} \mathrm{gal} / \mathrm{Mpc}^{3} / \mathrm{Gyr} / \log \mathcal{M}$ and is even lower approaching zero for higher masses. The growth rate in mass density is $\dot{\rho}_{\mathcal{M}} \simeq 10^{6}-10^{7} \mathcal{M}_{\odot} / \mathrm{Mpc}^{3} / \mathrm{Gyr} / \log \mathcal{M}$. Integrating above $\log \left(\mathcal{M} / \mathcal{M}_{\odot}\right)=9.8$, we find that $\dot{\rho}_{N}=6.8( \pm 1.2) \times$ $10^{-4} \mathrm{gal} / \mathrm{Mpc}^{3} / \mathrm{Gyr}$ and $\dot{\rho}_{\mathcal{M}}=1.8( \pm 0.4) \times 10^{7} \mathcal{M}_{\odot} / \mathrm{Mpc}^{3} / \mathrm{Gyr}$ between the second and the first redshift bin $(z \sim 0.34)$. Despite the large errors, we find a clear increase with cosmic time in the growth rate of galaxies with $\log \left(\mathcal{M} / \mathcal{M}_{\odot}\right)=$ 10.4-10.7, but a hint of a decrease with cosmic time at higher $\mathcal{M}\left(\log \left(\mathcal{M} / \mathcal{M}_{\odot}\right)=10.7-11\right)$. For $\log \left(\mathcal{M} / \mathcal{M}_{\odot}\right)>11$, the growth rate in number density is consistent with zero for almost all 


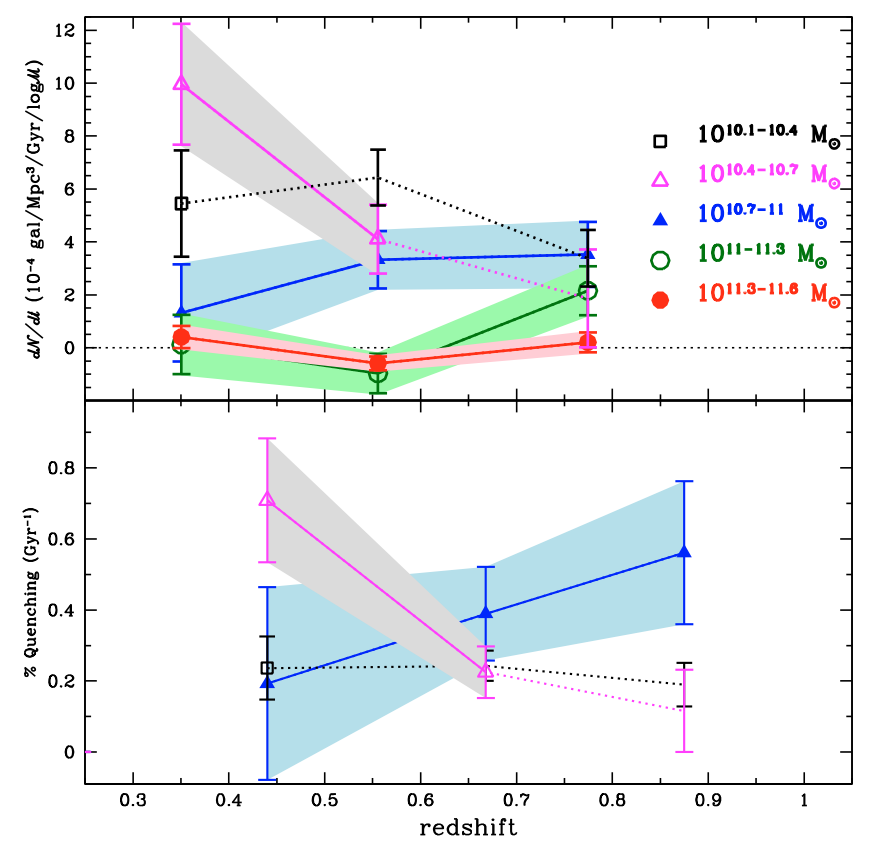

Fig. 15. Evolution of the growth rate in number density $\dot{\rho}_{N}$ (top panel) as a function of redshift for red galaxies $(\mathcal{P T}=1)$ in different mass ranges (represented by different symbols and colours). Values have been plotted at the intermediate redshift between two adjacent redshift bins. In the bottom panel, we show the evolution with redshift of the fraction of blue galaxies $(\mathcal{P T}=2-4)$ that are transforming into red galaxies per Gyr (see text). In all the panels, hatched regions refer to GSMFs that are complete in the mass range and redshift considered, while dotted lines are connecting points at redshifts where the GSMF is not complete in the considered mass range.

redshift bins. These trends can be interpreted as a mass-assembly downsizing signal, i.e., most massive red galaxies assembled their mass earlier than lower mass red galaxies. For comparison, Arnouts et al. (2007) for the VVDS found that the median mass growth rate (integrated over the entire mass range $\left.\log \left(\mathcal{M} / \mathcal{M}_{\odot}\right)=8-13\right)$ between $z \sim 2$ and $z=0$ is $1.7( \pm 0.4) \times$ $10^{7} \mathcal{M}_{\odot} / \mathrm{Mpc}^{3} / \mathrm{Gyr}$, which is consistent with our values for $\log \left(\mathcal{M} / \mathcal{M}_{\odot}\right)>9.8$. Walcher et al. (2008) derived a somewhat higher value of $\dot{\rho}_{\mathcal{M}}=6.5-10 \times 10^{7} \mathcal{M}_{\odot} / \mathrm{Mpc}^{3} / \mathrm{Gyr}$ from $z \sim 1$ to $z \sim 0.5$ integrated over the whole mass range. The difference from our results, indeed, could be due to their more extended mass range.

In principle, by comparing this growth rate with the number density of blue galaxies we should be able to estimate the fraction of blue galaxies that, at any given time, are transforming into red galaxies $\left(f_{Q}\right)$ after the quenching of their $S F R$. We therefore derived the fraction rate of quenching

$f_{\mathrm{Q}}(\mathcal{M})=\frac{\dot{\rho}_{N}^{\text {red }}(\mathcal{M})}{\rho_{N}^{\text {blue }}(\mathcal{M})} \mathrm{Gyr}^{-1}$,

using the $1 / V_{\max }$ estimation for $\rho_{N}$, as shown in the bottom panel of Fig. 15 as a function of redshift and mass. For $\log \left(\mathcal{M} / \mathcal{M}_{\odot}\right)>11$, the rates were unconstrained because of the low number density of blue galaxies. The values obtained for $\log \left(\mathcal{M} / \mathcal{M}_{\odot}\right)<11$ have a median value of $\sim 25 \% \mathrm{Gyr}^{-1}$. As for the growth rate, we find that with cosmic time this fraction rate decreases for high-mass galaxies, but increases for intermediatemass galaxies, at least for $z<0.7$. At $z<0.4$, we are unable to explore the transformation from blue to red for the low mass regime $\left(\log \left(\mathcal{M} / \mathcal{M}_{\odot}\right)<10.4\right)$ and to verify whether the trend of the blue fraction increasing with cosmic time for low-mass galaxies is shifted to even lower redshift, as expected by the downsizing scenario. We note however that all fraction values remain very uncertain and their uncertainties increase yet further if we include the effects of cosmic variance.

Vergani et al. (2009) explore the properties and number densities of the post-starburst (PSB) galaxy population, spectroscopically identified in the zCOSMOS sample, as the possible link population in the transition phase between the blue cloud and the red-sequence. They found that this galaxy population, which is affected by a sudden quenching of its star-formation activity, may increase the stellar mass density of the red-sequence by up to a non-negligible level of $10 \%$.

\subsection{Elliptical/early type galaxy evolution}

Hierarchical semi-analytical models (De Lucia et al. 2006) predict that stars in more massive elliptical galaxies, relative to their lower-mass counterparts, are older but assemble their mass by means of mergers later in cosmic time. For example, $50 \%$ of ellipticals with $\log \left(\mathcal{M} / \mathcal{M}_{\odot}\right)>11$ form most $(80 \%)$ of their stars at $z_{\text {form }} \simeq 1.6$, but assemble their mass by $z_{\text {assembly }} \simeq 0.2$, which is defined as the redshift at which most $(80 \%)$ of the stars that make up the galaxy at redshift zero are already assembled into a single object. Galaxies with $\log \left(\mathcal{M} / \mathcal{M}_{\odot}\right)>9.6$, instead, have a median $z_{\text {form }} \simeq 1.3$ and median $z_{\text {assembly }} \simeq 0.9$ (see Figs. 4 and 5 in De Lucia et al. 2006). As pointed out by Cimatti et al. (2006), this trend is the opposite of that of the mass-assembly downsizing found for red galaxies in the luminosity function, confirmed by Scarlata et al. (2007b), i.e., most massive red galaxies assembled their mass earlier than lower-mass red ones. In the following, we explore in detail for the zCOSMOS data set the evolution of elliptical and all early-type galaxies, using the different classification schemes described in Sect. 4 based on colours, morphology, spectral features, or a combination of them.

Figure 16 of the GSMFs for the different populations of ETGs: red galaxies $(\mathcal{P T}=1)$, passive galaxies $\left(\log \left(S S F R / \mathrm{Gyr}^{-1}\right)<-2\right)$, spheroidals $(\mathcal{M T}=1, \mathcal{Z} \mathcal{T}=1+2.0$, $\mathcal{Z} \mathcal{T}=1)$, absorption-line galaxies $(\mathcal{S} \mathcal{T}=1)$, and "bona fide ETGs" as defined in Sect. 4.

First of all, we note that there are significant differences in the normalization of the GSMFs at all $\mathcal{M}$, the pure ellipticals classified by ZEST $(\mathcal{Z T}=1)$ having the lowest value at all redshifts and mass ranges explored. In contrast, the shapes of the various GSMFs are all similar, there being a decline in number density for $\mathcal{M}<\mathcal{M}^{*}$ for all GSMFs that is described well by a single Schechter function, except for the GSMF of the spheroids with $\mathcal{M T}=1$, which shows an excess at low-mass $\left(\log \left(\mathcal{M} / \mathcal{M}_{\odot}\right)<9.5\right)$ in the first redshift bin.

We then studied in detail this population of low-mass spheroids $(\mathcal{M T}=1)$, which dominates the $G S M F$ and exhibits blue colours $\left(\mathcal{P T}>=2\right.$, i.e., $\left.(U-B)_{\text {rest }}<1\right)$. We found 89 of these objects with $\log \left(\mathcal{M} / \mathcal{M}_{\odot}\right)<9$ at low redshift $(z<0.5)$. They have quite compact sizes, with a small median effective radius $\left(r_{\mathrm{e}} \simeq 0.6 \mathrm{kpc}\right)$. We also identified a contribution from blue MRS spheroids $(\mathcal{P T}>=2, \mathcal{M T}=1)$ also at high mass $\left(\log \left(\mathcal{M} / \mathcal{M}_{\odot}\right)>10.5\right)$ at all redshifts $(182$ objects $)$. This latter population of massive blue MRS spheroids does not dominate the high-mass end of the GSMF $(\sim 20 \%)$, as the former does the low-mass end. Therefore, this suggests that the shape of the $G S M F$ for this population of blue spheroids differs from that for red spheroids, at least when using MRS morphologies. This is consistent with the conclusions of Zucca et al. (2009) about the contribution of this population to the $B$-band luminosity 


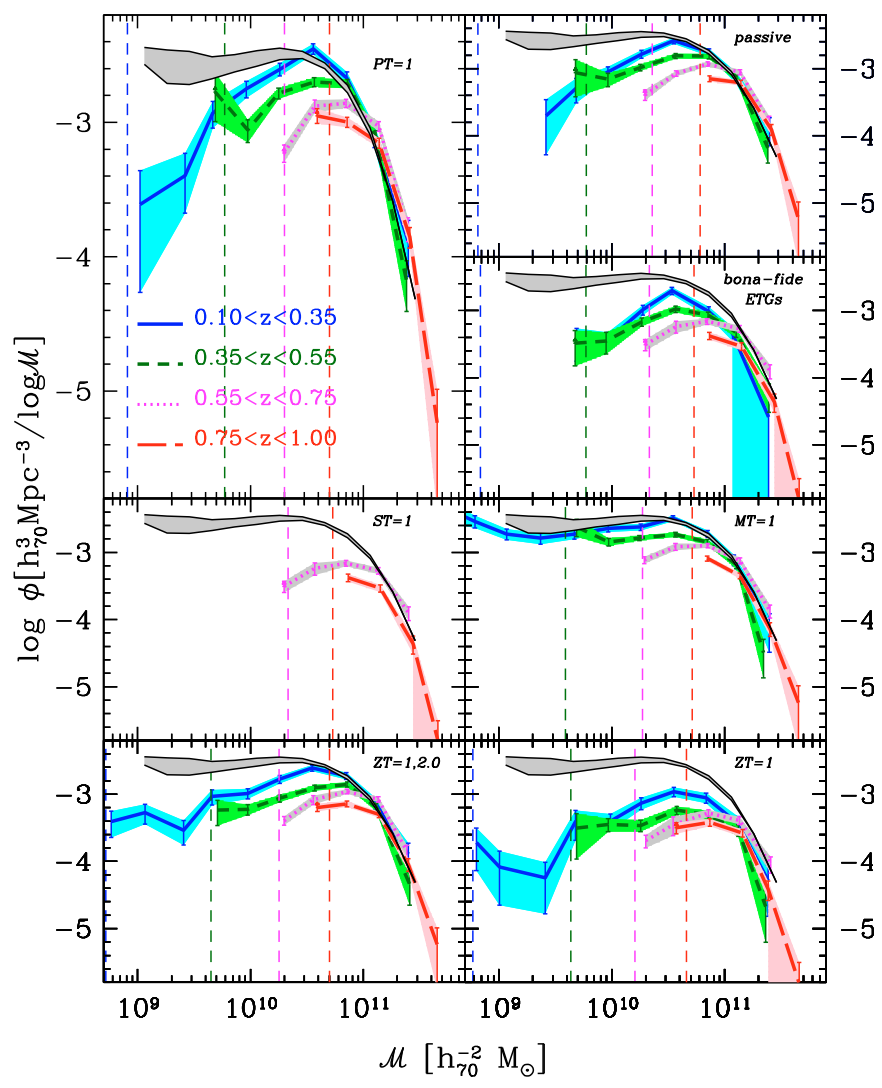

Fig. 16. $G S M F$ s for ETGs using various classification schemes: red galaxies $(\mathcal{P T}=1)$, passive $\left(\log \left(S S F R / \mathrm{Gyr}^{-1}\right)<-2\right)$, spheroidals $(\mathcal{Z T}=1+2.0, \mathcal{Z T}=1, \mathcal{M T}=1)$, absorption-line galaxies $(\mathcal{S T}=1)$, and "bona fide ETGs" as defined in Sect. 4. Only $1 / V_{\max }$ determinations have been plotted, with their uncertainties. Different lines and colours refer to different redshift ranges (see upper left panel). Grey hatched region, bounded by solid black lines, refer to local GSMF for ETGs derived from Baldry et al. $(2008,2006)$ (see text).

function. However, for these objects, in particular at low mass, we note that MRS morphologies do not always agree with ZEST ones, which implies that there are significant uncertainties in deriving morphologies for faint low-mass objects. We, therefore, verified their morphologies visually (i.e., PC, LT, LP). We found that most of the blue low-mass MRS spheroids are bulgedominated spirals (Sa). Most of the massive ones, even if their morphological classifications are in agreement between the two methods (ZEST and MRS), appear to be spirals, between Sa and Sc, although they have morphological parameters (asymmetry and concentration) that are more similar to those of elliptical galaxies. Composite spectra of this population were generated (following Mignoli et al. 2009) by averaging all their spectra at $z<0.5$ and dividing them into low-mass and high-mass objects. In Fig. 17, the average spectra are plotted: low-mass blue spheroids show a blue continuum and emission lines typical of star-forming galaxies, indicating that these galaxies are experiencing significant star-formation, while the high-mass blue spheroids have on average a red spectrum with absorption lines that are largely indistinguishable from those of the purely passive galaxies (see Mignoli et al. 2009), but in addition exhibit emission lines that could be indicative of some nuclear activity contamination (i.e., $[\mathrm{NII}] / \mathrm{H}_{\alpha}$ and $[\mathrm{OIII}] / \mathrm{H}_{\beta}$ are consistent with LINERs). An AGN component may also explain their typically high concentration index obtained from their HST images. These

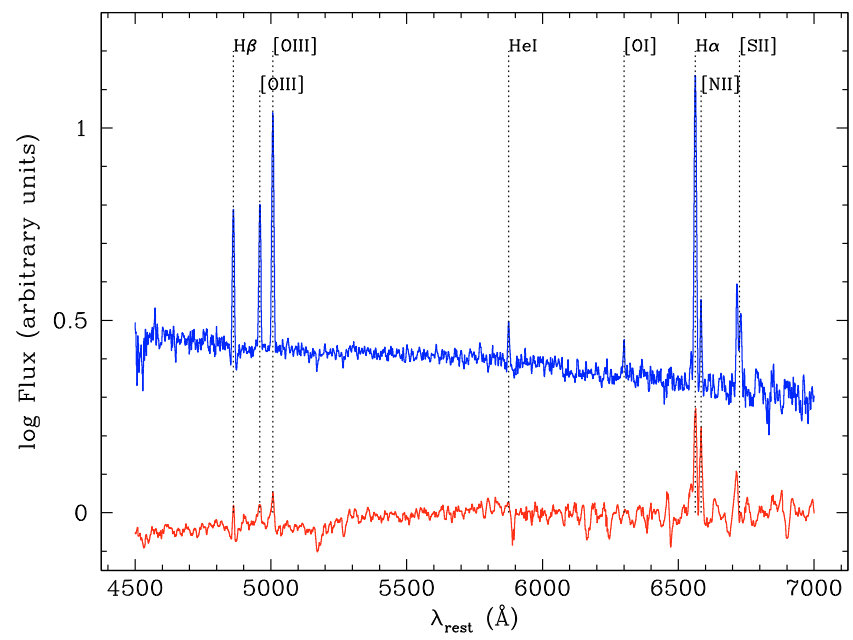

Fig. 17. Stacked spectra for galaxies with MRS elliptical morphology $(\mathcal{M T}=1)$ and blue colours $(\mathcal{P T}=2+3+4)$ at lowmass $\left(\log \left(\mathcal{M} / \mathcal{M}_{\odot}\right)<9\right.$ upper spectrum in blue) and high-mass $\left(\log \left(\mathcal{M} / \mathcal{M}_{\odot}\right)>10.5\right.$ lower spectrum in red).

AGN might play a role in quenching the $S F R$ in this population of blue spheroidal massive galaxies, which are possibly in the transition phase between the blue cloud and the red-sequence.

Finally, we studied the evolution with cosmic time of the GSMFs of each of the classes of red, passive, ellipticals, spheroids, absorption-line, and "bona fide ETG" galaxies. In Fig. 16, we show the GSMFs of these various ETG classes at increasing redshift. We also show, as reference, the local GSMF of ETGs, which we derived using the global GSMF by Baldry et al. (2008) and the fraction of red galaxies estimated by Baldry et al. (2006) for their 4 central environment bins. This is indicated in Fig. 16 by the grey shaded region. We note very similar evolutions in the ETG population for all the different means of classifying an ETG. As claimed for red galaxies, we find a clear "mass-assembly downsizing" evolution, with no evidence of an increase with cosmic time in the number density of the most massive ETGs since $z \sim 1$ and a progressive increase in the $G S M F$ s at intermediate mass $\left(\log \left(\mathcal{M} / \mathcal{M}_{\odot}\right)<11\right)$ out to $z=0$. Using M05 models to estimate the stellar masses, we also find that the same trend is visible in our data, and that the use of these models does not strongly affect our main conclusions. This is because in the redshift range studied here $(0.1<z<1.0)$, ETGs have on average old stellar populations that are not dominated by TP-AGB stars particularly in massive objects.

For massive ETGs, similar results were first noted in the near-IR ( $K$-band) luminosity function by Pozzetti et al. (2003) and the mass function by Fontana et al. (2004) in the K20 survey, using spectral classification, and later confirmed by larger optical and near-IR surveys, such as the VVDS, using colours or spectra to define ETGs (Zucca et al. 2007; Arnouts et al. 2007; Vergani et al. 2008). Scarlata et al. (2007b) found that in COSMOS both morphologically and photometrically selected subsamples of ETGs show no evolution in their number density at the bright end of the $B$-band luminosity function $(L>$ $\left.2.5 L^{*}\right)$ out to $z \sim 0.7$, and there is a deficit of a factor of about 2-3 of fainter ETGs over the same cosmic period, as also confirmed by Zucca et al. (2009) in the zCOSMOS sample. Ilbert et al. (2010), selecting ETGs (by morphology or colour) in SCOSMOS (Sanders et al. 2007) with accurate photometric redshifts (Ilbert et al. 2009) up to $z_{\text {photo }}=2$, found similar results for massive ETGs at $z<1$ (evolution $<0.2$ dex for 


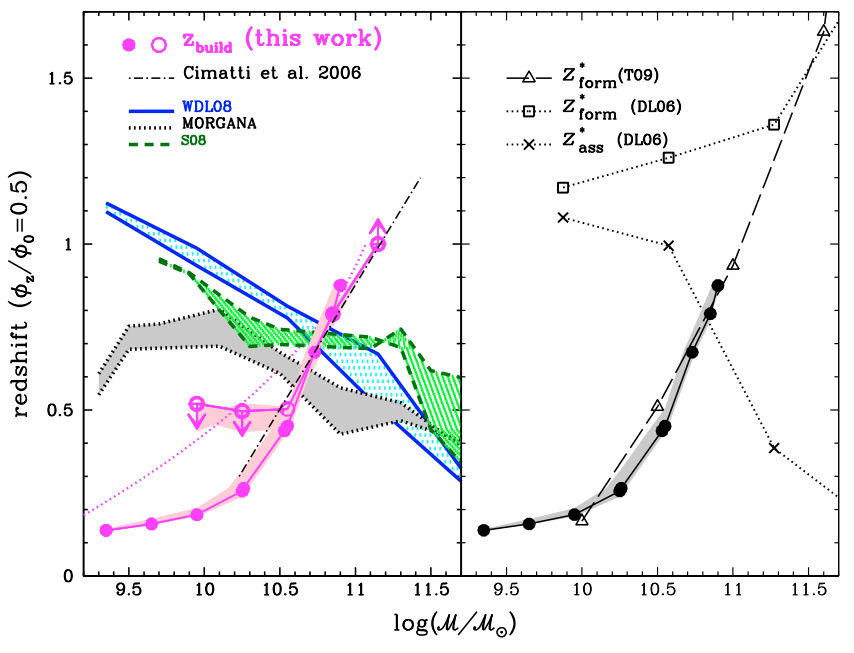

Fig. 18. Redshift at which the ETG GSMF has decreased by a factor 2 $\left(\phi_{z} / \phi_{0}=0.5\right.$, i.e., $z_{\text {building }}$, see text $)$ as a function of $\mathcal{M}$. Left panel: circles magenta points refer to data derived within zCOSMOS for passive galaxies $\left(\log \left(S S F R / \mathrm{Gyr}^{-1}\right)<-2\right)$ : open circles have been derived completely from zCOSMOS dataset, using as a reference the GSMF in the first redshift bin $\left(\phi_{0}=\phi(z \sim 0.24)\right.$, along with the region covered using various ETG classification criteria; filled circles are $z_{\text {building }}$ using the local ETG GSMF (see text), along with the spread in its shape related to their enviroment. Dotted magenta curve shows the $\mathcal{M}_{\text {min }}$ above which we are formally complete. Also plotted are zbuilding from SAMs: WDL08 (shaded region bounded by solid blue lines), S08 (shaded region bounded by dashed green lines), and MORGANA (shaded region bounded by dotted black lines), derived using the original GSMF or convolved with 0.25 dex in mass uncertainties (see Fontanot et al. 2009). Right panel: $z$ building for zCOSMOS data points are compared with the trend with stellar mass of the median $z_{\text {assembly }}$ and $z_{\text {form }}(80 \%$ of star assembled and formed, respectively) from De Lucia et al. (2006, DL06) and with the redshift at which the stellar formation ends ( $80 \%$ of star formed) in local ETGs estimated by Thomas et al. $(2009, \mathrm{~T} 09)$.

$\left.\log \left(\mathcal{M} / \mathcal{M}_{\odot}\right)>11\right)$ and a more rapid evolution at higher redshifts (by a factor of 15-20 between $z=1.5-2$ and $z=0.8-1$ ) or lower masses (increasing by a factor of 4.5 between $z=0.8-1$ and $z=0.2-0.4$ for $\left.\log \left(\mathcal{M} / \mathcal{M}_{\odot}\right) \sim 10\right)$.

\subsection{The building redshift of ETGs}

Our results suggest that lower mass ETGs (regardless of the method used to classify them) assembled their mass later than higher-mass ETGs. We can, indeed, estimate the redshift at which $50 \%$ of ETGs have already built-up their mass ( $z_{\text {building }}$ ), as a function of stellar mass, i.e., at which redshift their number density has decreased by a factor of 2 from $z=0\left(\phi_{z} / \phi_{0}=0.5\right)$. Since we consider the number densities of ETGs only above $z>0.25$, we can derive only upper limits to $z_{\text {building, }}$, as defined above, at least in the low mass range. For this reason, we used as a local reference the GSMF for ETGs, described in the previous section and shown in Fig. 16 as a grey shaded region.

Within the zCOSMOS redshift range, we find for ETG

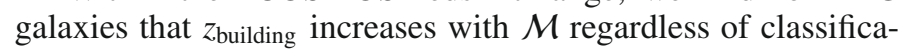
tion method, with $z_{\text {building }} \sim 0.4,0.5$, and 0.8 for $\log \left(\mathcal{M} / \mathcal{M}_{\odot}\right) \sim$ 10,10.4, and 10.8, respectively (Fig. 18). At higher mass, the number density of ETGs is almost constant up to redshift $\sim 0.7$ and decreases by less than a factor of 2 after $z \sim 1$, thus we set 1 as the lower limit to $z_{\text {building }}$ for $\log \left(\mathcal{M} / \mathcal{M}_{\odot}\right)>11$. Our estimates of $z_{\text {building }}$ for passive galaxies $\left(\log \left(S S F R / \mathrm{Gyr}^{-1}\right)<-2\right)$ are shown in Fig. 18: the open circle symbols were derived completely from the zCOSMOS dataset, using the GSMF in the first redshift bin $\left(\phi_{0}=\phi(z \sim 0.24)\right.$ as reference, and are shown along with the region covered using various ETG classification criteria. Most of the points have $\mathcal{M}>\mathcal{M}_{\min }$, above which we are formally complete. Since the local ETG GSMF, derived by Baldry et al. (2006, 2008) as described previously, approaches the observed zCOSMOS GSMF in the first redshift bin for $\log \left(\mathcal{M} / \mathcal{M}_{\odot}\right)>10.5$ for passive galaxies, below this mass the values of $z_{\text {building }}$ should be considered upper limits. We also show zbuilding derived by assuming the local GSMF for ETGs, along with the region covered using the different fractions of ETGs in the 4 central environment bins in Baldry et al. (2006). In addition, systematic uncertainties caused by the different classification methods could affect our estimates of $z_{\text {building, }}$ as for the zCOSMOS data.

First of all, we note that the "downsizing" trend with mass in terms of ETG $z_{\text {building }}$ is clearly visible. Our data are in very good agreement with the values derived by Cimatti et al. (2006), who used the de-evolved luminosity function for galaxies on the red-sequence. The values of $z_{\text {building }}$ are in quite close agreement with the end of the star formation ( $80 \%$ of the star formed) histories estimated by Thomas et al. (2009) for early-type galaxies as a function of stellar mass on the basis of their spectral properties (T09, in the right panel of Fig. 18). This downsizing trend with stellar mass conflicts instead with the prediction of semianalytical models for $z_{\text {assembly }}$ (e.g., by De Lucia et al. 2006, DL06, reproduced here in the right panel of Fig. 18). Given the increase with mass of the redshift at which the stars are predicted to form in this model ( $z_{\text {form }}$, see Fig. 18 right panel), the decreasing trend with mass of $z_{\text {assembly }}$ is caused by the increasing relevance of merging processes (in particular minor merging) with increasing mass (Wang \& Kauffmann 2008; Stewart et al. 2009; Parry et al. 2009) in the SAMs.

To perform a more accurate comparison with semi-analytical models (SAM), we used $z_{\text {building }}$ derived according to exactly the same definition applied to the data. We adopt the GSMF predictions for the three prescriptions used in Fontanot et al. (2009): the most recent implementation of the Munich SAM (De Lucia \& Blaizot 2007; Wang et al. 2008a, hereafter WDL08), the MORGANA model (Monaco et al. 2007; Lo Faro et al. 2009), and the fiducial model presented by Somerville (2008, hereafter S08). We used the same classification criteria for passive galaxies $\left(\log \left(S S F R / \mathrm{Gyr}^{-1}\right)<-2\right)$ as used in the models. The predicted $G S M F$ s for each model are shown in Fig. 6 of Fontanot et al. (2009). In Fig. 18, we compare $z_{\text {building }}$ derived from GSMF SAM predictions (provided by Fontanot) and zCOSMOS data using exactly the same definition. For each model, we show the region covered by $z_{\text {building }}$ using the original SAM GSMFs and those that take into account the $0.25 \mathrm{dex}$ of uncertainties in the mass derivation. In contrast to the data, the semi-analytical models show a reverse trend with mass of $z_{\text {building }}$, which is similar to the trend of $z_{\text {assembly. }}$ Residual small differences, for example between the determination of mass or SFR for the data and that of the models (e.g., the presence of secondary bursts in the model SFH) or in the ETG classification criteria are unlikely to reconcile the opposite trend with mass.

Taken at face value, the coincidence of $z_{\text {form }}$ estimated by Thomas et al. (2009) and $z_{\text {building }}$ argues against the dominant contribution of mergers (major+minor) at $z<1$ in building up ETG galaxies, as instead predicted by semi-analytical models (Wang \& Kauffmann 2008; Stewart et al. 2009). We therefore conclude that the build-up of ETG galaxies follows the same 
"downsizing" trend in mass as the formation of their stars, which disagrees with the "upsizing" trend predicted in SAMs.

\subsection{Timescales for the quenching of the star formation and morphological transformation}

The evolution with redshift in the galaxy number density in different mass ranges for three different samples of ETG (red $(\mathcal{P T}=1)$, spheroidal $(\mathcal{Z T}=1,2.0)$, and quiescent $\left.\left(\log \left(S S F R / \mathrm{Gyr}^{-1}\right)<-1\right)\right)$ galaxies is shown in Fig. 19. We find that they all show a very similar trend (but a different normalization), there being an increase in the number densities of intermediate-mass ETGs with cosmic time from $z=1$ to the local Universe. This trend is steep for low-tointermediate mass galaxies and is flat and almost negligible only for $\log \left(\mathcal{M} / \mathcal{M}_{\odot}\right)>11(<0.1$ dex between $z=0.85$ and $z=0.25$ for $\left.\log \left(\mathcal{M} / \mathcal{M}_{\odot}\right)=11-11.5\right)$. The similar evolutions of red and spheroidal galaxies was already noted by Arnouts et al. (2007), who commented: "if not by chance, this coincidence could suggest that the build-up of the quiescent sequence is closely followed or preceded by a morphological transformation". In principle, if all the various classification methods here adopted are "perfect", that the number density of SED-ETGs is higher than that of the morpho-ETGs, at a given mass, suggests that the colour transformation (from blue to red) precedes or has a shorter timescale than the morphological transformation. From Fig. 19, the delay time between the colour and the morphological transformation could be estimated, at a fixed $\rho_{N}$, to be about $1-2$ Gyr (as shown by the arrow for $\log \left(\mathcal{M} / \mathcal{M}_{\odot}\right)=10.7-11$ ). In addition, since the number density of the low-SSFR population is higher than that of the red galaxies (SED-ETGs), we estimate that the time elapsed for quiescent galaxies to completely switch-off their SFR and become red is of the same order (about 1-2 Gyr, shown as an arrow in Fig. 19). Wolf et al. (2008) reach a similar conclusion in the cluster A901/2 at $z \sim 0.17$ : the rich red-spiral population at intermediate mass $\left(\log \left(\mathcal{M} / \mathcal{M}_{\odot}\right)=[10,11]\right)$ is more accurately explained if quenching is a slow process and morphological transformation is delayed even more (see also Skibba et al. 2009). The small contribution to the assembly of the red-sequence by the class of post-starburst galaxies (most of which are assumed to quench their star formation rapidly) provides additional support to a scenario of delayed/slow quenching (Vergani et al. 2009).

However, we are aware that the colour-selected red galaxies may be contaminated by dusty starbursts. We recall here that, given our $24 \mu \mathrm{m}$ flux limit of $\sim 0.3 \mathrm{mJy}$, only $5 \%$ of red galaxies (SED-ETGs) were detected at $24 \mu \mathrm{m}$ (all having $\left.\log \left(S S F R / \mathrm{Gyr}^{-1}\right)>-2\right)$, and about $23 \%$ have $-2<$ $\log \left(\operatorname{SSFR} / \mathrm{Gyr}^{-1}\right)<-1$, i.e., they are not completely "dead". Bundy et al. 2010, through MIPS stacking analysis, show that the implied SFRs of red galaxies are on average at least an order of magnitude below the star-forming population. On the other hand, there is a non-negligible blue population $(\sim 20 \%)$ among elliptical galaxies, for which therefore the morphological transformation could precede or is faster than the colour transformation (see below for a discussion). Therefore, we also checked that the number density of red passive galaxies $\left(\mathcal{P T}=1\right.$ and $\left.\log \left(S S F R / \mathrm{Gyr}^{-1}\right)<-2\right)$, i.e., those that are "red and dead", is higher at any given mass and redshift than the number density of passive elliptical galaxies $(\mathcal{Z T}=1,2.0$ and $\left.\log \left(S S F R / \mathrm{Gyr}^{-1}\right)<-2\right)$, and that at a given number density and mass they are delayed by 1-2 Gyr. We conclude that, at least statistically, the morphological transformation takes a longer time than for a given galaxy to become "red and dead".

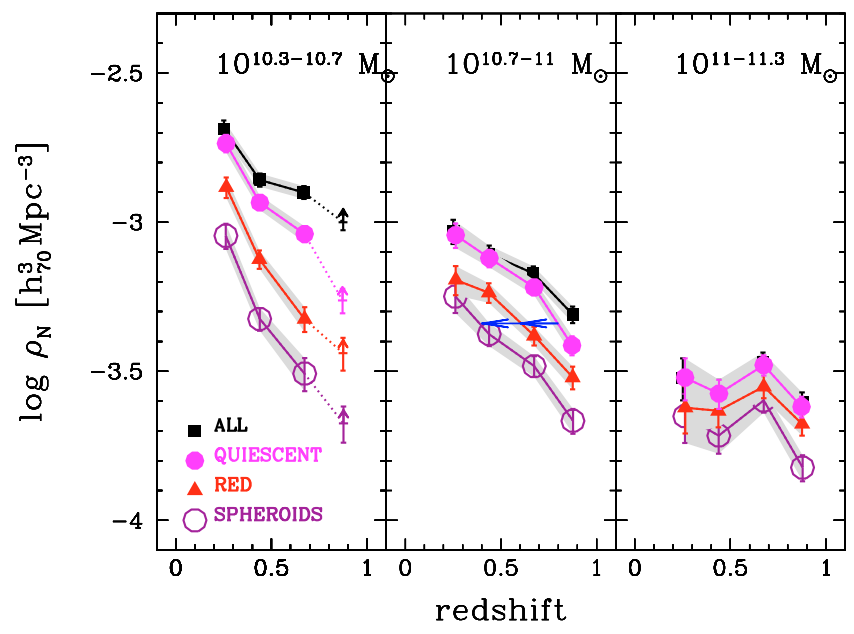

Fig. 19. Number density evolution for ETGs in different mass ranges: red galaxies (red filled triangles for $\mathcal{P} \mathcal{T}=1$ ), spheroidals (dark magenta empty circles for $\mathcal{Z T}=1+2.0$ ) and low-SSFR (magenta filled circles for $\log (S S F R)<-1)$. Black filled squares refer to the global population. Lines have the same meaning as in Fig. 11. In the second panel, we also indicate by 2 arrows the evolutionary time sequence from quiescent to red to spheroidal galaxies (see text).

But which physical processes induce these transformations? All the properties SSFR, colours, and photometric types depend on the star-formation history, while morphological types mainly reflect the dynamical history of a galaxy. The transformation from active blue galaxies to red passive galaxies, as well as the transformation from spiral disc-dominated to bulge-dominated systems, could be driven by a variety of processes such as the ageing of their stellar population or the fading of the disc, because of the exhaustion of its gas reservoir, gas stripping, or the quenching of the SFHs, for example by AGN feedback (Menci et al. 2006; Monaco et al. 2007; Bower et al. 2006), or by truncating the gas accretion from infall or from satellite galaxies. In addition, "starvation" (or "strangulation") is expected to affect a galaxy star formation history on a quite long timescale and therefore to cause a slowly declining activity: when a galaxy is accreted onto a larger structure, the gas supply can no longer be replenished by cooling, which is suppressed (Larson et al. 1980). This process is an important element of semi-analytic models of galaxy formation. Some of the aforementioned processes are also obviously environment-dependent.

On the other hand, the strong increase in the number density of intermediate-mass pure morphological ellipticals $(\mathcal{Z T}=1)$ or "bona fide ETGs" (red passive spheroidals) provides support for dynamical processes, such as the merging between two lowmass galaxies (Toomre \& Toomre 1972; Barnes 1992). Mergers are included in standard semi-analytic models of galaxy formation and represent indeed the main channel of formation for bulges. Menci et al. (2002) also included in their hierarchical semi-analytical models binary aggregations of satellite galaxies inside a common halo. However, if merger events were responsible for morphological transformations, we would expect to observe a rapid evolution from blue to red after the formation of the bulge/elliptical, i.e., after the burst triggered by the merger (Mihos \& Hernquist 1996). We find, instead, that the colour transformation precedes the morphological one, and that only a population $(\sim 20 \%)$ of massive blue spheroidal galaxies exists, which could be the end-product of merger events. The massive tail of this population has some signature of an AGN component in its stacked spectrum (see Fig. 17), which may be responsible 
L. Pozzetti et al.: zCOSMOS: Galaxy bimodality in the stellar mass function
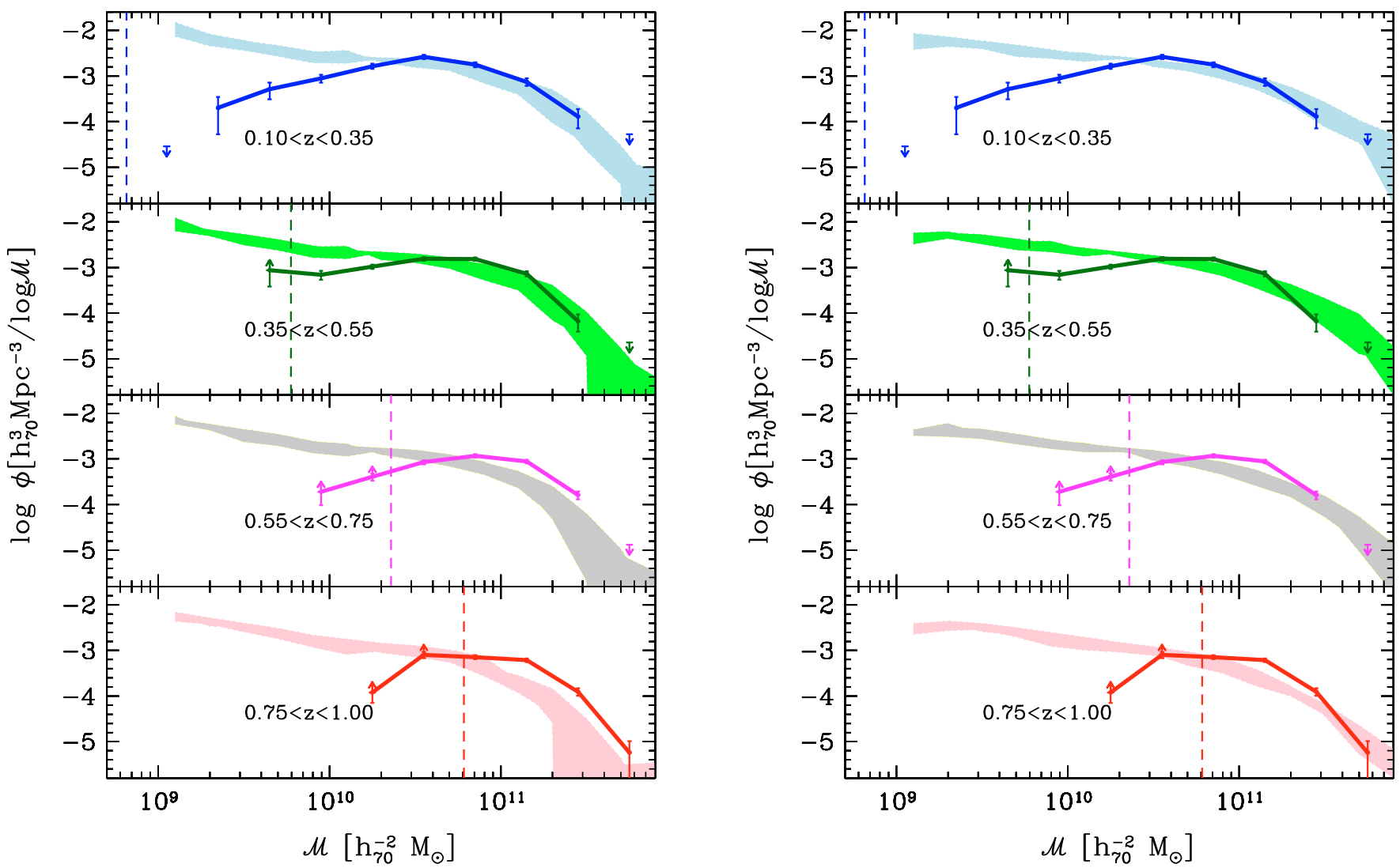

Fig. 20. Comparison between observed GSMFs (lines and points) with the regions covered by the predictions (shaded colour regions) of three semi-analytical models (Morgana, WDL08, and S08 from Fontanot et al. 2009), at increasing redshifts (from top to bottom panels). Left panels: original GSMFs from SAM. Right panels: SAM GSMFs take into account 0.25 dex of uncertainties in the $\mathcal{M}$ derivation.

for quenching the SFR, allowing their transition to red ellipticals with cosmic time. The study of this particular population is beyond the aim of this paper and will be the subject of future zCOSMOS papers.

Our results, therefore, suggest that dynamical processes, other than mergers, are the main mechanisms responsible for the morphological transformation that follows the colour transformation. For example, bulge growth through disc instability could be efficient (Carollo et al. 2001; Bower et al. 2006; Dekel et al. 2009). Disc instability is also a possible formation channel for bulges in SAMs (De Lucia et al. 2006; Bower et al. 2006; Parry et al. 2009). Dynamical observations of massive galaxies at $z \simeq 2$ with SINFONI (Genzel et al. 2006, 2008) also suggest that disc instability can produce massive bulges, even in the absence of major mergers. Indeed, dynamical friction and viscous processes at $z \simeq 2$ proceed on a timescale of $<1 \mathrm{Gyr}$, which is at least one order of magnitude faster than in $z \sim 0$ disc galaxies. In overdense environments, such as clusters, ram pressure may also play a role, demolishing the star-forming disc (Cortese et al. 2006). Recently, Bundy et al. (2010) demonstrated that the colour and morphological transformations should proceed through several separate stages and explored the strengths and weaknesses of several more sophisticated explanations, including environmental effects, internal stabilization, and disc regrowth by means of gas-rich mergers.

\section{Comparison with models}

To establish the cause of the "age-downsizing" of elliptical galaxies, according to which the stars in more massive galaxies formed earlier and over a shorter period than those in less massive galaxies, Cattaneo et al. (2008) discuss a model in which star formation shutdowns in dark matter haloes of mass above a critical value of $\sim 10^{12} \mathcal{M}_{\odot}$. However, this model predicts an evolution in the massive tail of the GSMF of red galaxies that is more rapid than that of intermediate-mass red galaxies from $z=1$ to $z=0$, which is in contrast, at least qualitatively, with our results (see Ilbert et al. 2010, for a comparison between model and data).

The same behaviour is clearly visible in Fig. 6 of Fontanot et al. (2009), which shows that the evolution of the GSMF for passive galaxies with redshift follows exactly the opposite trends with mass and cosmic time observed in the data (compare with our Fig. 16). They also conclude that a robust prediction of SAM models seems to be that the evolution of less massive galaxies is slower than for more massive ones, i.e., the models do not predict "mass-downsizing" but rather the opposite behaviour (sometimes called "upsizing"). Here we recall that the three semi-analytical model renditions considered in Fontanot et al. (2009) are indeed unable to reproduce the "mass-assembly downsizing" evolution of observed ETG GSMF, as parametrized for example by the median building redshift described in the previous section (Fig. 18).

In Fig. 20, we also show the direct comparison of zCOSMOS ETG GSMF with the SAM model predictions for passive galaxies $\left(\log \left(\operatorname{SSFR} / \mathrm{Gyr}^{-1}\right)<-1\right)$. Hatched region show the range covered by the three models from Fontanot et al. (2009) for the original GSMFs (left panel) and convolved with 0.25 dex uncertainties in the mass estimate (right panel). A more detailed and quantitative comparison between each model and data is beyond the aim of this paper. We first note that the shape of the predicted GSMFs differs from that of the observed 
GSMFs in all the mass and redshift ranges explored. In particular, the models are unable to recover the steep decline with decreasing mass in the number densities of passive ETGs, but instead overproduce their number densities increasingly with decreasing mass for $\log \left(\mathcal{M} / \mathcal{M}_{\odot}\right)<10.3$. This is because in the models, intermediate-mass galaxies form too early and are too passive and red at late times. In contrast, at the massive mass end $\left(\log \left(\mathcal{M} / \mathcal{M}_{\odot}\right)>11\right)$ the original models have the well-known problem of underestimating the number density of massive galaxies at high redshift (for $z>0.6$ in zCOSMOS dataset). We show that the convolution with stellar mass uncertainties $(0.25$ dex $)$ only partially solve the problem at high redshift and high masses $\left(\log \left(\mathcal{M} / \mathcal{M}_{\odot}\right)>11.3\right)$, while models continue to underpredict $G S M F$ s around $\log \left(\mathcal{M} / \mathcal{M}_{\odot}\right) \sim 11-11.3$, where they fail to reproduce the GSMF shape, and overproduce massive galaxies at low redshifts, as already pointed out by Fontanot et al. (2009). To qualitatively reconcile data and models at the massive-end, the mass uncertainties would have to increase rapidly with redshift, which is not the case for most current surveys with accurate and multi-band photometry that extends to near-IR rest-frame wavelengths.

We conclude that the three semi-analytical model explored here (WDL08, MORGANA, and S08) are unable to completely reproduce either the observed shape of the GSMFs of passive galaxies or the evolutionary trend. In particular, SAMs overpredict the low-mass ETG number densities and, even if the uncertainties in $\mathcal{M}$ determination partially account for the underprediction at high masses and high redshifts, they predict an evolution with cosmic time that is of an opposite trend with mass to that observed for the data, i.e., they are unable to reproduce the later formation and more rapid evolution in number densities of the lower mass galaxies ("mass-assembly downsizing").

\section{Summary and conclusions}

We have investigated the evolution of the galaxy stellar mass function up to $z=1$ in the zCOSMOS 10k bright spectroscopic survey using VIMOS spectroscopy $(\sim 8500$ galaxies with $15.0<$ $I<22.5$ over $1.4 \mathrm{deg}^{2}$ ) and multiband photometry (from UV to near-IR). Our main observational results can be summarized as follows:

- Unveiling bimodality in the GSMF: the shape of the GSMF is more accurately reproduced by two Schechter functions at least up to $z \simeq 0.55$, the maximum redshift at which we can explore the GSMF upturn due to our mass limit. This shape is linked to the bimodality in the galaxy properties of the ETG and LTG populations, which dominate the GSMF, respectively, at high masses $\left(\log \left(\mathcal{M} / \mathcal{M}_{\odot}\right)>10.5\right)$ and at low/intermediate-masses up to the highest redshift explored $(z \simeq 1)$.

- Mass-assembly downsizing: we find a continuous, rapid increase with cosmic time from $z \simeq 1$ to $z=0$ in the global $G S M F$ for $\log \left(\mathcal{M} / \mathcal{M}_{\odot}\right)<11$ (by a factor of $\sim 2$ for $\left.\log \left(\mathcal{M} / \mathcal{M}_{\odot}\right) \sim 10.5\right)$ and a more slow increase $(<15 \%)$ for $\log \left(\mathcal{M} / \mathcal{M}_{\odot}\right)>11$. A similar but even stronger trend is inferred for the ETG population, i.e., massive galaxies buildup their stars and mass earlier than lower mass galaxies. We show that the $z_{\text {building }}$ of ETGs increases rapidly with $\mathcal{M}$ from $z_{\text {building }} \sim 0.2$ to $\sim 0.8$ for $\log \left(\mathcal{M} / \mathcal{M}_{\odot}\right)=10,10.8$, respectively, and is significantly higher $(z \gtrsim 1)$ for massive ETGs.

- "SFH-evolved GSMF" and mergers: assuming only a growth in stellar mass driven by exponentially decreasing star-formation rates, we find that the predicted "SFH-evolved
GSMFs" agree with the observed GSMFs with differences of at most 20-40\%, even if they predict redder colours than observed, in particular at low redshift. Therefore, mass assembly is controlled primarily by SFH, which can explain most of the evolution of the GSMF at intermediate-low $\mathcal{M}$ $\left(\log \left(\mathcal{M} / \mathcal{M}_{\odot}\right)<10.6\right)$. The low residual evolution of the $G S M F$ is consistent with 0.16 merger per galaxy per Gyr, on average, with a hint of a decrease with cosmic time but no clear dependence on mass. Major merging events contribute in terms of fewer than $0.1 \mathrm{merger} / \mathrm{gal} / \mathrm{Gyr}(\sim 0.06$ on average) and are, if not marginal, not the dominant evolutionary process.

- $\mathcal{M}_{\text {cross }}$ and the evolution of the LTGs: the intersection $\left(\mathcal{M}_{\text {cross }}\right)$ between the GSMFs of the ETG and LTG populations decreases with cosmic time. This is due mainly to a clear increase with cosmic time in the number density of ETGs, regardless of their classification method, for $\log \left(\mathcal{M} / \mathcal{M}_{\odot}\right)<11$. In contrast, for LTGs the number density of blue or disc+irregular galaxies shows only a mild or negligible evolution, while the most extreme population of star-forming galaxies (those with high specific star formation) decreases rapidly in number density with cosmic time, in particular at high mass $\left(\log \left(\mathcal{M} / \mathcal{M}_{\odot}\right)>10.3\right)$.

- Flow to build-up the red population: we have quantified the flow rate from blue to red population at different redshifts and masses. We have found a growth rate in number and mass density of the red galaxies of about a few $10^{-4} \mathrm{gal} / \mathrm{Mpc}^{3} / \mathrm{Gyr} / \log \mathcal{M}$ and a few $10^{6}-10^{7} \mathcal{M}_{\odot} / \mathrm{Mpc}^{3} / \mathrm{Gyr} / \log \mathcal{M}$ for $\log \left(\mathcal{M} / \mathcal{M}_{\odot}\right)<11$, and lower in value above this mass. The corresponding fraction of blue galaxies that, at any given time, are transforming into red galaxies per Gyr is on average 25\% for $\log \left(\mathcal{M} / \mathcal{M}_{\odot}\right)<11$. This fraction and the growth rate for red galaxies increases with $\operatorname{cosmic}$ time at $\log \left(\mathcal{M} / \mathcal{M}_{\odot}\right)=$ $10.4-10.7$ at least for $z<0.7$, while it seems to decrease with cosmic time at higher $\mathcal{M}\left(\log \left(\mathcal{M} / \mathcal{M}_{\odot}\right)=10.7-11\right)$.

- ETG evolutionary sequence: using different methods to classify ETGs, we have found that the number density of quiescent galaxies is higher than that of red galaxies, which is even higher than that of spheroidals for $\log \left(\mathcal{M} / \mathcal{M}_{\odot}\right)<11$. This behaviour suggests that a transformation occurs from quiescent to red objects, as inferred mainly from SFHs, before or on a shorter timescales than the morphological tranformation, which is most likely primarily driven by dynamical processes.

- Comparison with SAM: semi-analytical models are unable to fully account for GSMF evolution, in particular for ETGs. They predict a different shape at any mass and redshift explored, there being a strong excess of low-mass passive galaxies and a deficit of high-mass galaxies at high$z$, disagreement that can be only partially resolved by the bias introduced by uncertainties in the mass determination. Furthermore, they predict an "upsizing" evolution in number density with cosmic time, i.e., mass assemply occurring slower and earlier in low-mass galaxies, which is the opposite of the "downsizing" trend observed in zCOSMOS data for the GSMF and quantified by $z_{\text {building. }}$.

We attempt to summarise our results within a galaxy evolutionary scenario that emerges from several observational studies. It is almost established that there is an age-downsizing of galaxy stellar populations, according to which less massive galaxies contain younger stars, which is also applicable to similar spectral/morphological types both among ETGs (Nelan et al. 2005; 
Thomas et al. 2005; Graves et al. 2007; Fontana et al. 2004) and LTGs (Noeske et al. 2007a,b). Furthermore, we find that downsizing is also applicable to the mass-assembly, i.e., massive galaxies assemble their mass in a single object earlier than low-mass galaxies, both for the global population and for ETGs, regardless of their definition (in terms of their colours, morphology, or SFR activity).

We therefore propose a scenario where intermediate-mass galaxies $\left(\log \left(\mathcal{M} / \mathcal{M}_{\odot}\right)=10-11\right)$ decrease their star formation activity to intermediate values gradually (with an e-folding time $\tau=1-3 \mathrm{Gyr}$ ) and finally to that of quiescent galaxies, by means of the exhaustion of either the gas reservoir or cold gas accretion, or quenching due to AGN feedback. Noeske et al. $(2007 \mathrm{a}, \mathrm{b})$ found that the e-folding time is mass-dependent, being longer for less massive galaxies, which also have a later onset of the SFH. Therefore, if no other mechanism intervenes to again switch on the star-formation activity (e.g., re-juvenation hypothesis, Hasinger et al. 2008), they evolve to have red colours on a timescale of 1-2 Gyr and finally undergo a dynamical morphological transformation into spheroidal galaxies.

In this scenario, massive galaxies $\left(\log \left(\mathcal{M} / \mathcal{M}_{\odot}\right)>11\right)$, in particular ETGs, are already in place at $z=1$, and their $G S M F$ exhibit negligible evolution thereafter. In contrast, the number density of intermediate-mass ETGs continues to increase with cosmic time (by a factor $\sim 4-2$ from $z=0.7$ to $z=0.2$ for $\left.\log \left(\mathcal{M} / \mathcal{M}_{\odot}\right)=10.3-10.7\right)$ with a growth rate of a few $10^{-4} \mathrm{gal} / \mathrm{Mpc}^{3} / \mathrm{Gyr} / \log \mathcal{M}$ and a few $10^{7} \mathcal{M}_{\odot} / \mathrm{Mpc}^{3} / \mathrm{Gyr} / \log \mathcal{M}$. We have found that the most likely evolutionary process to explain our results is the transformation from blue to red galaxies by means of the smooth decrease or the quenching of the SFR (as described above), followed by a morphological transformation probably by means of internal dynamical processes, such as disc instabilities. Major merger events are unlikely to be able to explain why colour transformation precedes the morphological one. In contrast, blue galaxies at low mass $\left(\log \left(\mathcal{M} / \mathcal{M}_{\odot}\right)<10\right)$ continue to grow in stellar mass, because of their high SSFR, and replace intermediate-mass LTGs, whose density remains almost constant in time. We have found that only a small fraction of low-mass $\left(\log \left(\mathcal{M} / \mathcal{M}_{\odot}\right) \sim 10\right)$ blue galaxies need to transform into red galaxies to explain the observed ETG growth rate at least at $z \geq 0.4$. Merger events (minor and major) contribute with a rate of $\sim 0.16 \mathrm{merger} / \mathrm{gal} / \mathrm{Gyr}$ on average (of which fewer than 0.1 are major) to form galaxies at redshifts lower than $z \sim 1$.

Following a schematic diagram, as originally proposed by Faber et al. (2007), this scenario is presented in Fig. 21. The dominant processes are the transformation with cosmic time of blue star-forming galaxies of progressively lower mass to red passive galaxies according to their SFH. Different evolutionary tracks are plotted between 0.1 and $10 \mathrm{Gyr}$ and refer to constraints on their inferred SFHs with different timescales (longer for less massive galaxies) and normalized to the total final mass $\left(\mathcal{M}_{\text {tot }}\right)$. As examples that only qualitatively reproduce our data, we show tracks for models with $\left(\tau ; \log \left(\mathcal{M}_{\text {tot }} / \mathcal{M}_{\odot}\right)\right)=$ $(0.1 ; 11.5),(0.3 ; 11.0)$ [solid red curves]; $(0.6 ; 10.7),(1 ; 10.3)$, $(2 ; 10.0)$ [short dashed green curves]; $(5 ; 11),(10 ; 10),(15 ; 9)$ [long dashed blue curves].

We conclude that the build-up of galaxies, and ETGs in particular, follows the same "downsizing" trend with mass (i.e., occurring earlier in high-mass galaxies) of the formation of their stars, which is the opposite of that predicted by current SAM models. The SAM LCDM models, with rapid galaxy growth, possibly overestimate the merger rate within halos (and underestimate the time-scale for merging). Our analysis

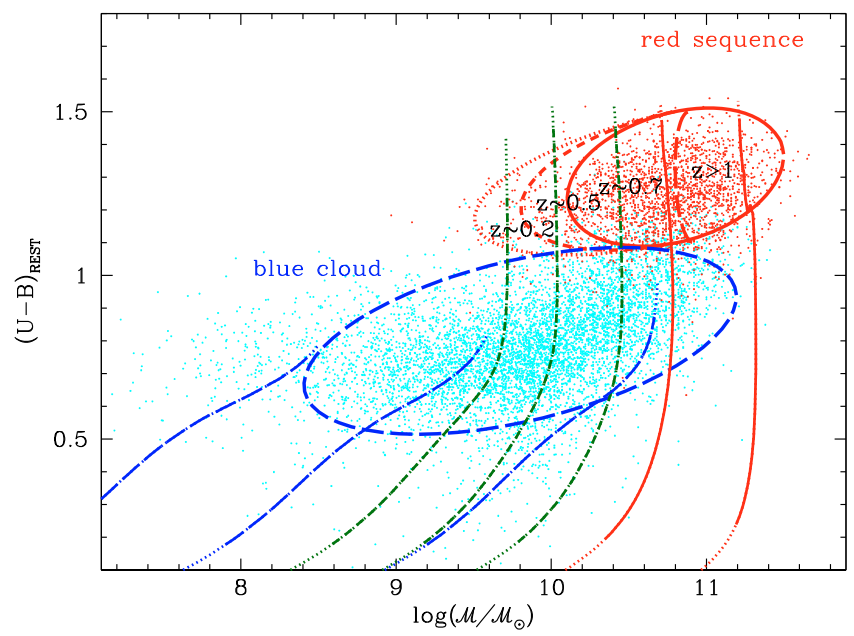

Fig. 21. Schematic scenario for galaxy evolution. Different ellipses refer to different redshifts and galaxy populations, while different evolutionary tracks are plotted between 0.1 and $10 \mathrm{Gyr}$ and refer to SFHs with different timescales and normalized to the total final mass $\left(\left(\tau ; \log \left(\mathcal{M}_{\text {tot }} / \mathcal{M}_{\odot}\right)\right)=(0.1 ; 11.5),(0.3 ; 11.0)\right.$ [solid red curves]; $(0.6 ; 10.7),(1 ; 10.3),(2 ; 10.0)$ [short dashed green curves]; $(5 ; 11)$, $(10 ; 10),(15 ; 9)$ [long dashed blue curves].

also suggests that morphological transformation should occur on longer timescales than colour transformation. Merging processes are not marginal but their frequency $(\sim 0.16$ merger/gal/Gyr of which $<0.1$ are major) appears to decrease with redshift, while no strong dependence on mass has been found, in disagreement with SAM models (Wang \& Kauffmann 2008; Stewart et al. 2009). The prevailing galaxy formation and evolutionary scenario is therefore moving towards one in which a smoother evolution in mass growth and star formation (due to accretion of cold gas) and minor merger play a more important role than major merging events. In this scenario, however, it appears quite difficult to explain the "fine-tuning" of the transformation of low-mass into intermediate-mass blue galaxies required to ensure that their number density remains almost constant at any given mass. In this scenario, we expect negligible evolution of the galaxy baryonic mass function $(G B M F)$ for the global population at all masses and a decrease with cosmic time in the $G B M F$ for the blue galaxy population at intermediate-to-high masses.

Acknowledgements. L.P. wish to thank Fabio Fontanot and Gabriella De Lucia for the stimulating discussions and to have provided SAM models and derived properties in electronic form. L.P. thanks Micheal Brown and Ramin Skibba for comments. We acknowledge support from an INAF contract PRIN2007/1.06.10.08 and an ASI grant ASI/COFIS/WP3110 I/026/07/0.

\section{References}

Abraham, R. G., van den Bergh, S., \& Nair, P. 2003, ApJ, 588, 218 Arnouts, S., Walcher, C. J., Le Fèvre, O., et al. 2007, 476, 137 Baldry, I. K., Glazebrook, K., Brinkmann, J., et al. 2004, ApJ, 600, 681 Baldry, I. K., Balogh, M. L., Bower, R. G., et al. 2006, MNRAS, 373, 469 Baldry, I. K., Glazebrook, K., Driver, S. P., et al. 2008, MNRAS, 388, 945 Balogh, M. L., Baldry, I. K., Nichol, R., et al. 2004, ApJ, 615, L101 Barnes, J. E. 1992, ApJ, 393, 484

Bauer, A. E., Drory, N., Hill, G. J., et al. 2005, ApJ, 621, L89

Bell, E. F., McIntosh, D. H., Katz, N., \& Weinberg, M. D. 2003, ApJ, 149, 289

Bell, E. F., Wolf, C., Meisenheimer, K., et al. 2004, ApJ, 608, 752

Bell, E. F., Phleps, S., Somerville, R. S., et al. 2006, ApJ, 652, 270

Bell, E. F., Zheng, X. Z., Papovich, C., et al. 2007, ApJ, 663, 834

Bertoldi, F., Carilli, C., Aravena, M., et al. 2007, ApJS, 172, 132

Blanton, M. R., Hogg, D. W., Bahcall, N. A., et al. 2003, ApJ, 594, 186

Blanton, M. R., Lupton, R. H., Schlegel, D. J., et al. 2005, ApJ, 631, 208 
Bolzonella, M., Miralles, J.-M., \& Pelló, R. 2000, A\&A, 363, 476 Bolzonella, M., Kovač, K., Pozzetti, L., et al. 2009, A\&A, in press [arXiv:0907.0013]

Borch, A., Meisenheimer, K., Bell, E. F., et al. 2006, A\&A, 453, 869

Bower, R. G., Benson, A. J., Malbon, R., et al. 2006, MNRAS, 370, 645 Brinchmann, J., \& Ellis, R. S. 2000, ApJ, 536, L77

Brinchmann, J., Charlot, S., White, S. D. M., et al. 2004, MNRAS, 351, 1151

Brown, M. J. I., Zheng, Z., White, M., et al. 2008, ApJ, 682, 937

Bruzual, G., \& Charlot, S. 2003, MNRAS, 344, 1000

Bundy, K., Ellis, R. S., Conselice, C. J., et al. 2006, ApJ, 651, 120

Bundy, K., Scarlata, C., Carollo, C. M., et al. 2010, ApJ, 719, 1969

Calzetti, D., Armus, L., Bohlin, R. C., et al. 2000, ApJ, 533, 682

Capak, P., Aussel, H., Ajiki, M., et al. 2007, ApJS, 172, 99

Caputi, K. I., McLure, R. J., Dunlop, J. S., et al. 2006, MNRAS, 366, 609Q

Carollo, C. M., Stiavelli, M., de Zeeuw, P. T., Seigar, M., \& Dejonghe, H. 2001, ApJ, 546, 216

Cassata, P., Guzzo, L., Franceschini, A., et al. 2007, ApJS, 172, 270

Cassata, P., Cimatti, A., Kurk, J., et al. 2008, A\&A, 483, L39

Cattaneo, A., Dekel, A., Faber, S. M., \& Guiderdoni, B. 2008, MNRAS, 389, 567

Chabrier, G. 2003, PASP, 115, 763

Charlot \& Bruzual 2007

Cimatti, A., Daddi, E., \& Renzini, A. 2006, A\&A, 453, L29

Cirasuolo, M., McLure, R. J., Dunlop, J. S., et al. 2007, MNRAS, 380, 585

Cole, S., Lacey, C. G., Baugh, C. M., \& Frenk, C. S. 2000, MNRAS, 319, 168

Cole, S., Norberg, P., Baugh, C. M., et al. 2001, MNRAS, 326, 255

Coleman, G. D., Wu, C.-C., \& Weedman, D. W. 1980, ApJS, 43, 393

Cortese, L., Gavazzi, G., Boselli, A., et al. 2006, A\&A, 453, 847

Cowie, L. L., Songaila, A., Hu, E. M., \& Cohen, J. G. 1996, AJ, 112, 839

Cucciati, O., Iovino, A., Marinoni, C., et al. 2006, A\&A, 458, 39

Dekel, A., Birnboim, Y., Engel, G., et al. 2009, Nature, 457, 451

De Lucia, G., \& Blaizot, J. 2007, MNRAS, 375, 2

De Lucia, G., Springel, V., White, S. D. M., Croton, D., \& Kauffmann, G. 2006, MNRAS, 366, 499

De Lucia, G., Poggianti, B. M., Aragón-Salamanca, A., et al. 2007, MNRAS, 374, 809

de Ravel, L., Le Fèvre, O., Tresse, L., et al. 2009, A\&A, 498, 379

Dickinson, M., Papovich, C., Ferguson, H. C., \& Budavári, T. 2003, ApJ, 587, 25

Driver, S. P., Phillipps, S., Davies, J. I., et al. 1994, MNRAS, 268, 393

Drory, N., Salvato, M., Gabasch, A., et al. 2005, ApJ, 619, L131

Drory, N., Bundy, K., Leauthaud, A., et al. 2009, ApJ, 707, 1595

Elvis, M., Civano, F., Vignali, C., et al. 2009, ApJS, 184, 158

Faber, S. M., Willmer, C. N. A., Wolf, C., et al., 2007, ApJ, 665, 265

Feldmann, R., Carollo, C. M., Porciani, C., et al. 2006, MNRAS, 372, 565

Felten, J. E. 1976, ApJ, 207, 700

Feulner, G., Gabasch, A., Salvato, M., et al. 2005a, ApJ, 633, L9

Feulner, G., Goranova, Y., Drory, N., et al. 2005b, MNRAS, 358, L1

Fontana, A., Pozzetti, L., Donnarumma, I., et al. 2004, A\&A, 424, 23, F04

Fontana, A., Salimbeni, S., Grazian, A., et al. 2006, A\&A, 459, 745

Fontanot, F., De Lucia, G., Monaco, P., Somerville, R. S., \& Santini, P. 2009, MNRAS, 397, 1776

Franzetti, P., Scodeggio, M., Garilli, B., et al. 2007, A\&A, 465, 711

Gavazzi, G., \& Scodeggio, M. 1996, A\&A, 312, L29

Gehrels, N. 1986, ApJ, 303, 336

Genzel, R., Tacconi, L. J., Eisenhauer, F., et al. 2006, Nature, 442, 786

Genzel, R., Burkert, A., Bouché, N., et al. 2008, ApJ, 687, 59

Giallongo, E., Salimbeni, S., Menci, N., et al. 2005, ApJ, 622, 116

Graves, G. J., Faber, S. M., Schiavon, R. P., \& Yan, R. 2007, ApJ, 671, 243

Gwyn, S. D. J., \& Hartwick, F. D. A. 2005, AJ, 130, 1337

Juneau, S., Glazebrook K., Crampton, D., et al. 2005

Hasinger, G., Cappelluti, N., Brunner, H., et al. 2007, ApJS, 172, 29

Hogg, D. W., Blanton, M., Strateva, I., et al. 2002, AJ, 124, 646

Hopkins, A. M., \& Beacom, J. F. 2006, ApJ, 652, 864

Ilbert, O., Arnouts, S., McCracken, H. J., et al. 2006, A\&A, 457, 841

Ilbert, O., Capak, P., Salvato, M., et al. 2009, ApJ, 690, 1236

Ilbert, O., Salvato, M., Le Floc'h, E., et al. 2010, ApJ, 709, 644

Iovino, A., Cucciati, O., Scodeggio, M., et al. 2010, A\&A, 509, 40

Kauffmann, G., Heckman, T. M., White, S. D. M., et al. 2003, MNRAS, 341, 33

Kennicutt, R. C. Jr. 1998, ARA\&A, 36, 189

Kitzbichler, M. G., \& White, S. D. M. 2007, MNRAS, 376, 2

Kinney, A. L., Calzetti, D., Bohlin, R. C., et al. 1996, ApJ, 467, 38

Kodama, T., Yamada, T., Akiyama, M., et al. 2004, MNRAS, 350, 1005

Koekemoer, A. M., Aussel, H., Calzetti, D., et al. 2007, ApJS, 172, 196

Kovač, K., Lilly, S. J., Knobel, C., et al. 2010a, ApJ, 718, 86

Kovač, K., Lilly, S. J., Cucciati, O., et al. 2010b, ApJ, 708, 505
Larson, R. B., Tinsley, B. M., \& Caldwell, C. N. 1980, ApJ, 237, 692

Le Fèvre, O., Saisse, M., Mancini, D., et al. 2003, SPIE, 4841, 1670

Lilly, S. J., Le Fevre, O., Hammer, F., \& Crampton, D. 1996, ApJ, 460, L1

Lilly, S. J., Le Fèvre, O., Renzini, A., et al. 2007, ApJS, 172, 70

Lilly, S. J., Le Brun, V., Maier, C., et al. 2009, ApJS, 184, 218

Lin, L., Koo, D. C., Willmer, C. N. A., et al. 2004, ApJ, 617, L9

Lo Faro, B., Monaco, P., Fontanot, F., et al. 2009, MNRAS, 399, L827

Madau, P., Ferguson, H. C., Dickinson, M. E., et al. 1996, MNRAS, 283, 1388

Madau, P., Pozzetti, L., \& Dickinson, M. 1998, ApJ, 498, 106

Maier, C., Lilly, S. J., Zamorani, G., et al. 2009, ApJ, 694, 1099

Maraston, C. 2005, MNRAS, 362, 799

Maraston, C., Daddi, E., Renzini, A., et al. 2006, ApJ, 652, 85

Marchesini, D., van Dokkum, P. G., Forster Schreiber, N. M., et al. 2009, ApJ, 701, 1765

Menci, N., Fontana, A., Giallongo, E., et al. 2005, ApJ, 632, 49

Menci, N., Fontana, A., Giallongo, E., et al. 2006, ApJ, 647, 753

Meneux, B., Guzzo, L., de la Torre, S., et al. 2009, A\&A, 505, 463

McCracken, H. J., Capak, P., Salvato, M., et al. 2010, ApJ, 708, 202

Mignoli, M., Zamorani, G., Scodeggio, M., et al. 2009, A\&A, 493, 39

Mihos, J. C., \& Hernquist, L. 1996, ApJ, 464, 641

Monaco, P., Murante, G., Borgani, S., \& Fontanot, F. 2006, ApJ, 652, L89

Monaco, P., Fontanot, F., \& Taffoni, G. 2007, MNRAS, 375, 1189

Moresco, M., Pozzetti, L., Cimatti, A., et al. 2010, A\&A, in press

Moustakas, J., Kennicutt, R. C., Jr., \& Tremonti, C. A. 2006, ApJ, 642, 775

Nelan, J. E., Smith, R. J., Hudson, M. J., et al. 2005, ApJ, 632, 137

Noeske, K. G., Faber, S. M., Weiner, B. J., et al. 2007a, ApJ, 660, L47

Noeske, K. G., Weiner, B. J., Faber, S. M., et al. 2007b, ApJ, 660, L43

Norberg, P., Cole, S., Baugh, C. M., et al. 2002, MNRAS, 336, 907

Oesch, P. A., Carollo, C. M., Feldmann, R., et al. 2010, ApJ, 714, L47

Parry, O. H., Eke, V. R., \& Frenk, C. S. 2009, MNRAS, 396, 1972

Popesso, P., Biviano, A., Bohringer, H., \& Romaniello, M. 2006, A\&A, 445, 29

Pozzetti, L., \& Mannucci, F. 2000, MNRAS, 317, L17

Pozzetti, L., Cimatti, A., Zamorani, G., et al. 2003, A\&A, 402, 837

Pozzetti, L., Bolzonella, M., Lamareille, F., et al. 2007, A\&A, 474, 443

Renzini, A. 2007, in At the Edge of the Universe: Latest Results from the

Deepest Astronomical Surveys, ed. J. Afonso, ASP Conf. Ser., 380, 309

Renzini, A. 2009, MNRAS, 398, L58

Salpeter, E. E. 1955, ApJ, 121, 161

Sandage, A., Tammann, G. A., \& Yahil, A. 1979, ApJ, 232, 352

Sanders, D. B., Salvato, M., Aussel, H., et al. 2007, ApJS, 172, 86

Sargent, M. T., Carollo, C. M., Lilly, S. J., et al. 2007, ApJS, 172, 434

Scarlata, C., Carollo, C. M., Lilly, S. J., et al. 2007a, ApJS, 172, 406

Scarlata, C., Carollo, C. M., Lilly, S. J., et al. 2007b, ApJS, 172, 494

Schechter, P. 1976, ApJ, 203, 297

Schinnerer, E., Smolčić, V., Carilli, C. L., et al. 2007, ApJS, 172, 46

Scodeggio, M., Franzetti, P., Garilli, B., et al. 2005, PASP, 117, 1284

Scoville, N., Aussel, H., Brusa, M., et al. 2007, ApJS, 172, 1

Schmidt, M. 1959, ApJ, 129, 243

Schmidt, M. 1968, ApJ, 151, 393

Skibba, R. A., Bamford, S. P., Nichol, R. C., et al. 2009, MNRAS, 399, 966

Somerville, R. S., Hopkins, P. F., Cox, T. J., Robertson, B. E., \& Hernquist, L. 2008, MNRAS, 391, 481

Stewart, K. R., Bullock, J. S., Barton, E. J., \& Wechsler, R. H. 2009, ApJ, 702, 1005

Strateva, I., Ivezic, Z., Knapp, G. R., et al. 2001, AJ, 122, 1861

Taniguchi, Y., Scoville, N., Murayama, T., et al. 2007, ApJS, 172, 9

Tasca, L., Kneib, J. P., Iovino, A., et al. 2009, A\&A, 503, 379

Thomas, D., Maraston, C., Bender, R., \& Mendes de Oliveira, C. 2005, ApJ, 621, 673

Thomas, D., Maraston, C., Schawinski, K., Sarzi, M., \& Silk, J. 2010, MNRAS, 404, 1775

Toomre, A., \& Toomre, J. 1972, ApJ, 178, 623

van Dokkum, P. G. 2005, AJ, 130, 2647

Vergani, D., Scodeggio, M., Pozzetti, L., et al. 2008, A\&A, 487, 89

Vergani, D., Zamorani, G., Lilly, S., et al. 2009, A\&A, 509, 42

Walcher, C. J., Lamareille, F., Vergani, D., et al. 2008, A\&A, 491, 713

Wang, L., \& Kauffmann, G. 2008, MNRAS, 391, 785

Wang, J., De Lucia, G., Kitzbichler, M. G., \& White, S. D. M. 2008, MNRAS, 384,1301

Weiner, B. J., Phillips, A. C., Faber, S. M., et al. 2005, ApJ, 620, 595

Williams, R. J., Quadri, R. F., Franx, M., van Dokkum, P., \& Labbé, I. 2009, ApJ, 691, 1879

Zamojski, M. A., Schiminovich, D., Rich, R. M., et al. 2007, ApJS, 172, 468

Zucca, E., Zamorani, G., Vettolani, G., et al. 1997, A\&A, 326, 477

Zucca, E., Ilbert, O., Bardelli, S., et al. 2006, A\&A, 455, 879

Zucca, E., Bolzonella, M., Bardelli, S., et al. 2009, A\&A, 508, 1217 
1 INAF - Osservatorio Astronomico di Bologna, via Ranzani 1, 40127 Bologna, Italy

e-mail: lucia.pozzetti@oabo.inaf.it

2 Institute of Astronomy, Swiss Federal Institute of Technology (ETH Hönggerberg), 8093 Zürich, Switzerland

3 Dipartimento di Astronomia, Universitá di Padova, Padova, Italy

4 Dipartimento di Astronomia, Universitá degli Studi di Bologna, via Ranzani 1, 40127 Bologna, Italy

5 Department of Astronomy, University of Massachusetts, 710 North Pleasant Street, Amherst, MA 01003, USA

${ }^{6}$ Laboratoire d'Astrophysique de Marseille, Université d'AixMarseille, CNRS, 38 rue Frederic Joliot-Curie, 13388 Marseille Cedex 13, France

7 INAF - Istituto di Astrofisica Spaziale e Fisica Cosmica di Milano, via Bassini 15, 20133 Milano, Italy

${ }^{8}$ Laboratoire d'Astrophysique de Toulouse-Tarbes, Université de Toulouse, CNRS, 14 avenue Édouard Belin, 31400 Toulouse, France
9 Max Planck Institut für Extraterrestrische Physik, Giessenbachstrasse, 84571 Garching, Germany

${ }^{10}$ Universitats Sternwarte, Scheinerstrasse 1, 81679 Muenchen, Germany

11 INAF - Osservatorio Astrofisico di Arcetri, Largo Enrico Fermi 5, 50125 Firenze, Italy

12 INAF - Osservatorio Astronomico di Brera, via Brera 28, 20121 Milano, Italy

13 Space Telescope Science Institute, 3700 San Martin Drive, Baltimore, MD 21218, USA

${ }_{15}$ Centre de Physique Théorique, Marseille, Marseille, France

15 Institut d'Astrophysique de Paris, Université Pierre \& Marie Curie, Paris, France

16 INAF - Osservatorio Astronomico di Roma, via di Frascati 33, 00040 Monteporzio Catone, Italy

17 Spitzer Science Center, Pasadena, CA, USA

18 California Institute of Technology, MC 105-24, 1200 East California Boulevard, Pasadena, CA 91125, USA 